\title{
Reversal of paclitaxel resistance in human ovarian cancer cells with redox-responsive micelles consisting of $\alpha$-tocopheryl succinate-based polyphosphoester copolymers
}

\author{
Feng-qian $\mathrm{CHEN}^{1, \#}$, Jin-ming ZHANG ${ }^{1,4, \#}$, Xie-fan FANG ${ }^{2}$, Hua YU ${ }^{1}$, Yu-ling LIU $^{3}$, Hui LI ${ }^{3}$, Yi-tao WANG ${ }^{1}$, Mei-wan CHEN ${ }^{1, *}$ \\ ${ }^{1}$ State Key Laboratory of Quality Research in Chinese Medicine, Institute of Chinese Medical Sciences, University of Macau, Macau, \\ China; ${ }^{2}$ Department of Pediatrics, College of Medicine, University of Florida, Gainesville, FL 32610, USA; ${ }^{3}$ Institute of Chinese Materia \\ Medica, China Academy of Chinese Medical Science, Beijing 100700, China; ${ }^{4}$ College of Pharmacy, Chengdu University of Traditional \\ Chinese Medicine, Chengdu 611137, China
}

\begin{abstract}
P-glycoprotein (P-gp)-mediated multidrug resistance (MDR) is a major obstacle in achieving the therapeutic benefits of paclitaxel (PTX) in the treatment of human ovarian carcinoma. This study is aimed to develop an efficient PTX drug delivery approach to overcome MDR. Redox-responsive micelles consisting of amphiphilic polymers containing disulfide linkages, ie, poly (phosphate ester)-SS-D- $\alpha$ tocopheryl succinate (POPEA-SS-TOS, PSST) were prepared. PTX-loaded PSST micelles (PTX/PSST-M) designed to display synergistic functions, including reversible inhibition of P-gp, intracellular redox-sensitive release and potent anticancer activities. The average size of PTX/PSST-M was $68.1 \pm 4.9 \mathrm{~nm}$. The encapsulated PTX was released quickly through redox-triggered dissociation of micelles. The inhibition of P-gp activity and enhanced cellular accumulation of the PSST micelles were validated. PTX/PSST-M showed significantly increased cytotoxicity against PTX-resistant human ovarian cancer A2780/PTX cells: when the cells were treated with PTX/PSST-M for $48 \mathrm{~h}$, the equivalent $\mathrm{IC}_{50}$ value of PTX was reduced from 61.51 to $0.49 \mu \mathrm{mol} / \mathrm{L}$. The enhanced cytotoxic effects of PTX/PSST-M against A2780/PTX cells were attributed to their synergistic effects on reducing the mitochondrial transmembrane potential, ATP depletion, ROS production, and activation of apoptotic pathways. Furthermore, PTX/PSST-M significantly increased cell apoptosis/necrosis and cell cycle arrest at the $\mathrm{G}_{2} / \mathrm{M}$ phase in A2780/PTX cells. These results demonstrate that the redox-responsive PSST micelles inhibit P-gp activity and have a good potential to effectively reverse PTX resistance in human ovarian carcinoma cells by activating intrinsic apoptotic pathways.
\end{abstract}

Keywords: ovarian carcinoma; paclitaxel; multidrug resistance; nanomedicines; D- $\alpha$-tocopheryl succinate; redox-responsive micelles; P-gp; apoptosis; cell cycle arrest; A2780/PTX cells

Acta Pharmacologica Sinica (2017) 38: 859-873; doi: 10.1038/aps.2016.150; published online 6 Mar 2017

\section{Introduction}

The morbidity and mortality of human ovarian carcinoma are rising worldwide. The therapeutic outcome of paclitaxel (PTX), a frontline chemotherapeutic agent for ovarian carcinoma ${ }^{[1]}$, is severely impaired due to the frequent occurrence of multidrug resistance $(\mathrm{MDR})^{[2]}$. It has been estimated that approximately 500000 new ovarian carcinoma cases develop MDR each year ${ }^{[3]}$. In particular, PTX resistance occurs in more

\footnotetext{
\# These authors contributed equally to this work.

${ }^{*}$ To whom correspondence should be addressed.

E-mail mwchen@umac.mo

Received 2016-08-04 Accepted 2016-10-12
}

than $70 \%$ of patients at the time of initial diagnosis and in almost all patients upon relapse ${ }^{[4]}$. The occurrence of MDR is an important cause of the failure of PTX to treat ovarian carcinoma. The potential mechanisms associated with MDR can be classified as acquired and intrinsic resistance. In particular, the overexpression of P-glycoprotein (P-gp), a member of the ATP-binding cassette (ABC) transporters that facilitate drug efflux, is the major mechanism of acquired resistance to drug treatment ${ }^{[5-7]}$. In addition, suppression of the apoptotic pathway is another important mechanism that induces $\mathrm{MDR}^{[8]}$. Therefore, reducing $\mathrm{P}$-gp levels and activating intrinsic apoptotic pathways may effectively overcome MDR.

For over two decades, the combination of chemotherapeu- 
tic agents with MDR modulators has been thought to be an effective strategy to overcome MDR. However, the major limitations of this strategy include potential toxicity caused by high concentrations of MDR modulators, such as verapamil, cyclosporin $\mathrm{A}^{[9]}$, and tariquidar ${ }^{[10]}$, and their unfavorable pharmacokinetic interactions with the therapeutic drugs. Recently, pharmaceutical excipients, including D-a tocopheryl polyethylene glycol 1000 succinate (TPGS) ${ }^{[11,12]}$ and D-a-tocopheryl succinate $(\mathrm{TOS})^{[13,14]}$, have been recognized by their potent inhibitory effects on P-gp by reducing the ATPase activity of P-gp. Considering their low bioactivity and toxicity, the application of copolymers conjugated with a TOS moiety to overcome MDR has gained increasing interest ${ }^{[13,15-18]}$. These TOS moiety-based nanomedicines exhibit desirable drug delivery characteristics such as high stability, high drug-loading capacity, prolonged systemic circulation lifetime, enhanced cellular uptake, and suppression of MDR by inhibiting P-gp.

These nanoparticles (NPs) are also faced with the common challenges encountered by other nano-vehicles ${ }^{[19,20]}$, including high hydrophobicity and a long degradation period, sometimes weeks or months. The slow degradation severely delays the release of cargo drugs in NPs, and the sufficient therapeutic drug concentration cannot be easily reached. To address this challenge, stimuli-responsive delivery systems that are sensitive to the aberrant intracellular environment, including abnormal $\mathrm{pH}$, redox property, and enzyme levels, have been developed to overcome MDR ${ }^{[21]}$. The glutathione (GSH) concentration gradient is one of the several stimuli utilized to trigger the intracellular drug delivery of $\mathrm{NPs}^{[22-24]}$. The GSH concentration is approximately $2-20 \mu \mathrm{mol} / \mathrm{L}$ in the extracellular milieu and $2-10 \mathrm{mmol} / \mathrm{L}$ in the intracellular fluids. In many reports, redox-responsive NPs consisting of disulfide bondlinking block copolymers have been demonstrated to enhance drug accumulation and retention in cells, leading to higher cytotoxicity against cancer cells with $\operatorname{MDR}^{[17,25,26]}$.

Recently, poly(phosphoesters) (PPEs) have been used in increasing biomedical applications as the burgeoning hydrophilic chains for drug and gene delivery due to their easy functionalization, favorable biocompatibility, and optimal biodegradability ${ }^{[27-29]}$. In this study, we aimed to develop an amphiphilic block copolyester, poly(phosphoesters)-g-TOS conjugated with disulfide bonds (POPEA-SS-TOS, PSST) via a Michael-type addition reaction. After validating the amphiphilicity and inhibitory activity of the PSST polymers against P-gp, PTX-loaded PSST micelles (PTX/PSST-M) were prepared by using a modified solvent evaporation method. The cellular uptake profiles and cytotoxicity of these shell-detachable micelles against PTX-sensitive A2780 cells and PTXresistant A2780/PTX cells were investigated. Furthermore, the mechanisms governing how these micelles reduced MDR were studied.

\section{Materials and methods} Materials

2-Chloro-2-oxo-1,3,2-dioxaphospholane (COP), 2-hydroxyethyl acrylate (HEA), D-a-tocopheryl succinate (TOS), cysta- mine dihydrochloride, triethylamine (TEA), and rhodamine 123 were purchased from Sigma-Aldrich (St Louis, MO, USA). Tetrahydrofuran (THF) was initially dried over molecular sieves (4A) for at least three days and refluxed over sodium wire until the indicator benzophenone turned purple. Carbodiimide (EDC) and N-hydroxysuccinimide (NHS) were obtained from GL Biochem (Shanghai, China). PTX (purity $>98 \%$ ) was obtained from Spring \& Autumn Biological Engineering (Nanjing, China). Hoechst 33342 and 3-(4,5-dimethylthiazol-2-yl)-2,5-diphenyltetrazolium bromide (MTT) were purchased from Biosharp (Seoul, South Korea). Penicillinstreptomycin (PS), 2' $7^{\prime}$-dichlorodihydrofluorescein diacetate (DCFH-DA), fetal bovine serum (FBS), trypsin without ethylene diamine tetraacetic acid (EDTA), phosphate-buffered saline (PBS), $0.25 \%$ trypsin $(w / v)$ with $1 \mathrm{mmol} / \mathrm{L}$ EDTA, and DMEM culture medium were purchased from Gibco (Grand Island, NY, USA).

\section{Cell culture}

The drug-sensitive human ovarian carcinoma A2780 cell line and the PTX-resistant A2780 cell line (A2780/PTX) were obtained from the American Type Culture Collection (ATCC, Manassas, VA, USA). The cells were cultured in DMEM containing $10 \%$ FBS and $2 \mathrm{mmol} / \mathrm{L} \mathrm{L}$-glutamine at $37^{\circ} \mathrm{C}$ in a humidified $5 \% \mathrm{CO}_{2}$ incubator.

\section{Synthesis and characterization of PSST polymer}

PSST polymer was obtained via a three-step synthesis process. i) TOS was conjugated with cystamine via an amidation reaction between the terminal carboxyl group and the amino group. Briefly, TEA (12 mmol) was added to the DMSO solution containing $5 \mathrm{mmol}$ cystamine dihydrochloride. The mixture was stirred for $2 \mathrm{~h}$ to remove hydrochloride and to generate cystamine. Subsequently, TOS (5 mmol), DCC (12 $\mathrm{mmol})$, and DMAP (12 mmol) were dissolved in the cystamine solution and maintained at $40^{\circ} \mathrm{C}$ for $6 \mathrm{~h}$. The TOS-SS-NH crude product was dialyzed in a dialysis bag (MWCO 500 $\mathrm{Da}$ ) against DMSO and then against distilled water to remove small molecules. The flaxen powder was obtained after lyophilization. ii) The monomer 2-(2-oxo-1,3,2-dioxaphospholoyloxy) ethyl acrylate (OPEA) was synthesized by an acyl halide reaction between COP and HEA in the presence of TEA, as described $^{[15,18]}$. The yield of OPEA was $73 \%$. iii) Finally, PSST polymer with a similar structure of pendants on a long chain was synthesized via ring-opening polymerization of OPEA in anhydrous THF using TOS-SS- $\mathrm{NH}_{2}$ as the macroinitiator and $\mathrm{Sn}(\mathrm{Oct})_{2}$ as the catalyst. To synthesize POPEA-SS-TOS, TOSSS- $\mathrm{NH}_{2}(1 \mathrm{mmol})$ and OPEA $(10 \mathrm{mmol})$ were dissolved in anhydrous THF in a 25-mL freshly dried and nitrogen-purged flask and stirred at room temperature for $15 \mathrm{~min}$. Sn(Oct) (0.5 mmol) was added, and the mixture was stirred for $12 \mathrm{~h}$ under nitrogen protection. The mixture was precipitated into excess cold diethyl ether, and PSST polymer was obtained by vacuum drying. The yield of POPEA-SS-TOS was 78.6\%.

The ${ }^{1} \mathrm{H}$ NMR spectra of the copolymers were obtained using a Varian Inova 400 spectrometer (Bruker Company, Billerica, 
MA, USA) operating at $400 \mathrm{MHz}$ with deuterated chloroform $\left(\mathrm{CDCl}_{3}\right)$ as the solvent. The molecular weight of the PSST polymer was determined by gel permeation chromatography (GPC) with THF as the mobile phase. The critical micellar concentration (CMC) value was obtained using a fluorescence method using pyrene as a fluorescent probe ${ }^{[30]}$. Additionally, the effects of the PSST polymer on MDR and P-gp expression were evaluated as described in the Supplementary information.

\section{Preparation of PTX-loaded PSST micelles (PTX/PSST-M)}

PTX/PSST-M were prepared by the solvent evaporation method $^{[31]}$. Briefly, $10 \mathrm{mg}$ PSST copolymer and $1.1 \mathrm{mg}$ PTX were dissolved in $1 \mathrm{~mL}$ methanol and dropwise added to $10 \mathrm{~mL}$ PBS (pH 7.4) with a syringe. The mixture was subsequently sonicated by a probe sonicator (Q700, Qsonica, Australia) for $1 \mathrm{~min}$ at $100 \mathrm{~Hz}$ and stirred for $2 \mathrm{~h}$ to become homodisperse. The residual organic solvent was eliminated by rotary vacuum evaporation at $40^{\circ} \mathrm{C}$ for $20 \mathrm{~min}$. To remove unloaded PTX and to maintain sterility, the micellar suspension was filtered through $0.45-\mu \mathrm{m}$ and $0.22-\mu \mathrm{m}$ filter membranes. Blank PSST micelles were prepared by the same procedures but without adding PTX. The size distribution and zeta potential of the micelles were detected by dynamic light scattering (DLS) at $25^{\circ} \mathrm{C}$ using a Zetasizer Nano ZSP (Malvern Instruments, Malvern, UK). Imaging of micelles stained with $1 \%$ phosphotungstic acid solution was performed with a transmission electron microscope (TEM, JEOL JEM-2100 F, Japan) at an accelerating voltage of $200 \mathrm{kV}$.

The PTX content in the micelles was measured by HPLC (Waters e2695, Milford, MA, USA) at $227 \mathrm{~nm}$ with acetonitrile/water $(70 / 30, v / v)$ as the mobile phase. The encapsulation efficiency (EE) and loading efficiency (LE) of the PTX in micelles were determined using the following equations:

$$
\begin{gathered}
E E(\%)=\frac{\text { Amount of drug loaded }}{\text { Amount of drug fed }} \times 100 \% \\
L E(\%)=\frac{\text { Amount of drug loaded }}{\text { Amount of drug fed polymer }} \times 100 \%
\end{gathered}
$$

\section{Reduction-triggered disassembly of the PSST micelles}

To evaluate the redox-sensitive behavior of the PSST micelles, the size change of micelles $(1 \mathrm{mg} / \mathrm{mL})$ in response to 10 mmol/L DTT was detected by DLS. Additionally, the selfassembly of PSST polymer was characterized by assessing the fluorescence spectra using Nile red (NR), a redox-insensitive but polarity-responsive hydrophobic dye, as the fluorescent probe. The amphiphilic aggregates loaded with NR were encapsulated into the micellar core via a hydrophobic interaction. Redox-triggered dissociation of PSST micelles was analyzed following the previously described procedure ${ }^{[32,33]}$. The fluorescence emission of the amphiphilic aggregates loaded with NR in an environment with or without $10 \mathrm{mmol} / \mathrm{L}$ DTT was examined via fluorescence spectrophotometry (Thermo Fisher Scientific, Waltham, MA, USA) with excitation and emission wavelengths of $550 \mathrm{~nm}$ and $500-800 \mathrm{~nm}$, respectively ${ }^{[34]}$.

\section{Controlled PTX release in vitro}

The in vitro release kinetics of PTX from the micelles was investigated as previously reported ${ }^{[35]}$. The dialysis tube method was employed, and PBS buffers (0.01 mol/L, pH 7.4) with or without $10 \mathrm{mmol} / \mathrm{L}$ DTT were used as the release medium. One milliliter of PTX/PSST-M was transferred to a dialysis bag (MWCO 3500) against $20 \mathrm{~mL}$ of release medium. Similarly, free PTX was dispersed in $0.1 \%$ Tween 80 and dialyzed in the same manner. The dialysis was conducted at $37^{\circ} \mathrm{C}$ with a rotation speed of 100 rounds per minute. At predetermined time-points, $1 \mathrm{~mL}$ release medium was sampled, and the release medium was replenished with the same volume of fresh medium. The PTX concentrations in the release medium were quantified by HPLC, as described above.

\section{Cellular uptake of PSST micelles in A2780/PTX cells}

A2780/PTX cells were seeded on 24-well culture plates at a density of $8 \times 10^{4}$ cells per well. After incubation for $24 \mathrm{~h}$, free PTX or PTX/PSST-M was added with an equivalent PTX concentration of $5 \mu \mathrm{mol} / \mathrm{L}$. The same treatments were carried out in A2780 cells as controls. After incubation for $0.5,1,2$, and 4 $\mathrm{h}$, the cells were washed twice with cold PBS and lysed with $1 \%$ Triton $\mathrm{X}-100$ at $37^{\circ} \mathrm{C}$ for $30 \mathrm{~min}$. The PTX concentrations in the cell lysates were measured by HPLC. The protein concentrations in the cell lysates were measured using a BCA Protein Assay kit (Thermo Fisher Scientific, USA). The intracellular PTX concentrations were normalized to the total protein content of the cell lysates.

To visualize cellular accumulation of drugs loaded in the PSST micelles, the fluorescent probe rhodamine 123 (Rho123), a P-gp substrate similar to PTX, was loaded into the PSST micelles to yield Rho-123-loaded PSST micelles (Rho-123/ PSST-M $)^{[36,37]}$. After treating A2780/PTX cells with free Rho123 or Rho-123/PSST-M at an equivalent Rho-123 concentration of $1 \mu \mathrm{mol} / \mathrm{L}$ for $0.5,1,2$, and $4 \mathrm{~h}$, the auto fluorescence of Rho-123 was measured using a flow cytometer (FCM, Thermo Fisher Scientific, USA) by counting 10000 events per sample.

In addition, the cellular accumulation of free Rho-123 and Rho-123/PSST-M in the A2780/PTX cells was observed by fluorescence microscopy. Briefly, A2780/PTX cells were seeded at a density of $5 \times 10^{3}$ cells/well in a 96-well plate. After adherence for $24 \mathrm{~h}$, the cells were treated with free Rho-123 or Rho-123/PSST-M at an equivalent Rho-123 concentration of $1 \mathrm{mmol} / \mathrm{L}$. The cells were washed three times with cold PBS, fixed with $4 \%$ paraformaldehyde for $15 \mathrm{~min}$, and stained with Hoechst 33342 for $10 \mathrm{~min}$. Fluorescence images were taken by an IN Cell Analyzer 2000 (GE Healthcare, Little Chalfont, UK).

To investigate the uptake mechanisms of the PSST micelles, A2780/PTX cells were pre-incubated with known transmembrane inhibitors for $1 \mathrm{~h}^{[38]}$. These inhibitors included $20 \mathrm{mmol} / \mathrm{L} 2$-deoxyglucose, $10 \mu \mathrm{mol} / \mathrm{L}$ chlorpromazine, 20 $\mu \mathrm{mol} / \mathrm{L}$ hexamethylene amiloride, $10 \mu \mathrm{mol} / \mathrm{L}$ wortmannin, $50 \mu \mathrm{mol} / \mathrm{L}$ genistein, and $5 \mathrm{mmol} / \mathrm{L}$ methyl- $\beta$-cyclodextrin. Next, the cells were treated with Rho-123/PSST-M at a concentration equivalent to $1 \mu \mathrm{mol} / \mathrm{L}$ Rho-123 for $4 \mathrm{~h}$. The cells were washed and analyzed by FCM. 


\section{Cytotoxicity of PTX formulations measured by MTT assay}

The in vitro cytotoxicity of PTX/PSST-M against A2780/PTX cells was determined via an MTT assay. Briefly, A2780 or A2780/PTX cells were seeded on 96-well plates at a density of $5 \times 10^{3}$ cell/well and cultured overnight. Free PTX, a physical mixture of free PTX and blank micelles (PTX+PSST-M), and PTX/PSST-M at a series of PTX concentrations were added to the cells. Untreated cells were used as control. After treatment for $48 \mathrm{~h}$ and $72 \mathrm{~h}$, cell viability was determined based on an MTT assay, as previously described ${ }^{[39]}$. All treatment groups had four replicates, and each replicate was measured three times.

\section{Measurement of mitochondrial transmembrane potential}

A2780/PTX cells were seeded on 12-well plates at a density of $1.0 \times 10^{5}$ cells per well. After $24 \mathrm{~h}$ of cell attachment, the cells were incubated with free PTX, PTX+PSST-M, or PTX/PSST-M at an equivalent PTX concentration of $1 \mu \mathrm{mol} / \mathrm{L}$ or at a polymer concentration of $100 \mu \mathrm{g} / \mathrm{mL}$ for $48 \mathrm{~h}$. Untreated cells acted as controls. Cells were examined with a JC-1 Mitochondrial Transmembrane Potential Assay kit (Beyotime Institute of Biotechnology, Haimen, China) according to the manufacturer's protocol. The mitochondrial transmembrane potential $\left(\Delta \psi_{\mathrm{m}}\right)$ was observed with a fluorescence microscope and quantitatively measured by FCM.

Quantification of intracellular ATP activity and reactive oxygen species (ROS)

A luciferin/luciferase assay was used to determine the intracellular ATP level ${ }^{[40,41]}$. Briefly, A2780/PTX cells were seeded on 12-well plates and treated with free PTX, PTX+PSST-M, or PTX/PSST-M at an equivalent PTX concentration of $1 \mu \mathrm{mol} / \mathrm{L}$ for $48 \mathrm{~h}$. Untreated cells acted as controls. Cells were lysed with 1\% Triton X-100, and ATP levels in the cell lysates were measured using an ATP Luminescence Assay kit (Beyotime, Haimen, China). The ATP levels were normalized to the protein content in the cell lysates, as determined by using a BCA Protein Assay kit.

Intracellular ROS was measured based on the oxidative conversion of DCFH-DA to a fluorescent compound, dichlorofluorescein $(\mathrm{DCF})^{[42]}$. A2780/PTX cells were treated with free PTX, PTX+PSST-M, or PTX/PSST-M at an equivalent PTX concentration of $1 \mu \mathrm{mol} / \mathrm{L}$ for $48 \mathrm{~h}$. Untreated cells acted as controls. The cells were washed and incubated with fresh medium containing $5 \mu \mathrm{mol} / \mathrm{L}$ DCHF-DA for $30 \mathrm{~min}$ in the dark. Intracellular ROS levels were measured with a FCM by detecting the green fluorescence of DCF.

Quantification of cell cycle arrest, apoptosis, and caspase 3/7 activity

Cell cycle arrest in A2780/PTX cells treated by various PTX formulations was analyzed by measuring the DNA content via the propidium iodide (PI) staining method. A2780/PTX cells were harvested after treatment with free PTX, PTX+PSST-M, or PTX/PSST-M at an equivalent PTX concentration of $1 \mu \mathrm{mol} / \mathrm{L}$ for $48 \mathrm{~h}$. Untreated cells acted as controls. The cells were fixed with $70 \%$ ethanol at $-20^{\circ} \mathrm{C}$ overnight and stained with 20 $\mathrm{mg} / \mathrm{mL}$ PI, which was dissolved in PBS containing $5 \mathrm{mg} / \mathrm{mL}$ RNase, for $30 \mathrm{~min}$. Samples were analyzed by FCM. The percentage of cells at each cell cycle phase was analyzed using ModFit software (Verity Software House, Topsham, ME, USA).

In addition, an Annexin V-FITC/PI double staining assay was used to detect apoptosis and necrosis of A2780/PTX cells after treatment with free PTX, PTX+PSST-M, or PTX/PSST-M at an equivalent PTX concentration of $1 \mu \mathrm{mol} / \mathrm{L}$ for $48 \mathrm{~h}$. Cells were harvested, suspended in $500 \mu \mathrm{L}$ binding buffer, and stained with $5 \mu \mathrm{L}$ Annexin-FITC and $5 \mu \mathrm{L}$ PI solution for 20 min in the dark. Untreated cells acted as controls. Cell samples were analyzed immediately by FCM, and 10000 events were counted for each sample.

Caspase 3/7 activity was measured using a Caspase-glo 3/7 Assay kit (Promega, Madison, WI, USA). After treatment with various PTX formulations at an equivalent PTX concentration of $1 \mu \mathrm{mol} / \mathrm{L}$ for $48 \mathrm{~h}, \mathrm{~A} 2780 / \mathrm{PTX}$ cells were incubated with Caspase-glo reagent for $1 \mathrm{~h}$ in the dark, and luminescence was measured using a microplate reader (Molecular Devices, USA). The luminescence signals were normalized to the number of cells determined from a parallel set of cells for each treatment.

\section{Statistical analysis}

The data were analyzed by one-way ANOVA followed by Tukey's Multiple Comparison Test using GraphPad Prism 5.0 software (La Jolla, CA, USA). $P<0.05$ was considered as statistically significant. The data were presented as the mean \pm standard deviation (SD).

\section{Results}

Synthesis and characterization of PSST polymer

The amphiphilic PSST polymer with a pendant VES moiety in the polyphosphoester block via disulfide linkages was synthesized by ring-opening polymerization (ROP), as shown in Scheme 1. The chemical structure of PSST was validated by ${ }^{1} \mathrm{H}$ NMR (Figure 1A). Notably, all the typical proton signals on the polymer were identified. The peaks within $\delta 0.8-1.8$ ppm were derived from the methyl and methylene groups in the tailed carbon chain of VES. The peak at $\delta 2.62 \mathrm{ppm}$ (a) represented the succinyl methylene in $\mathrm{VES}^{[43]}$. The cystamine linkage was confirmed by peaks b, c, and $d$ at $\delta 2.92$, 3.65 , and 4.76 ppm, respectively ${ }^{[44]}$. The methylene in the poly(phosphoester) chain appeared as peak e at $\delta 4.31 \mathrm{ppm}$. Additionally, peaks $g$ and $f$ were generated from the acryloyl group in the poly(phosphoester). The typical GPC overlay of the PSST polymer treated with or without $10 \mathrm{mmol} / \mathrm{L}$ DTT for $6 \mathrm{~h}$ is shown in Figure 1B. The PSST polymer was degraded into polymers with lower molecular weights ranging from approximately 5100 to 9800 , contributing to the redoxresponsive separation of VES from the poly(phosphoester) chain. Due to the amphiphilic structure, the newly synthesized PSST copolymer can easily form micelles in an aqueous solution by self-assembly. To determine the CMC value of the PSST polymer, the fluorescence intensity $\left(I_{374} / I_{358}\right)$ ratio of 


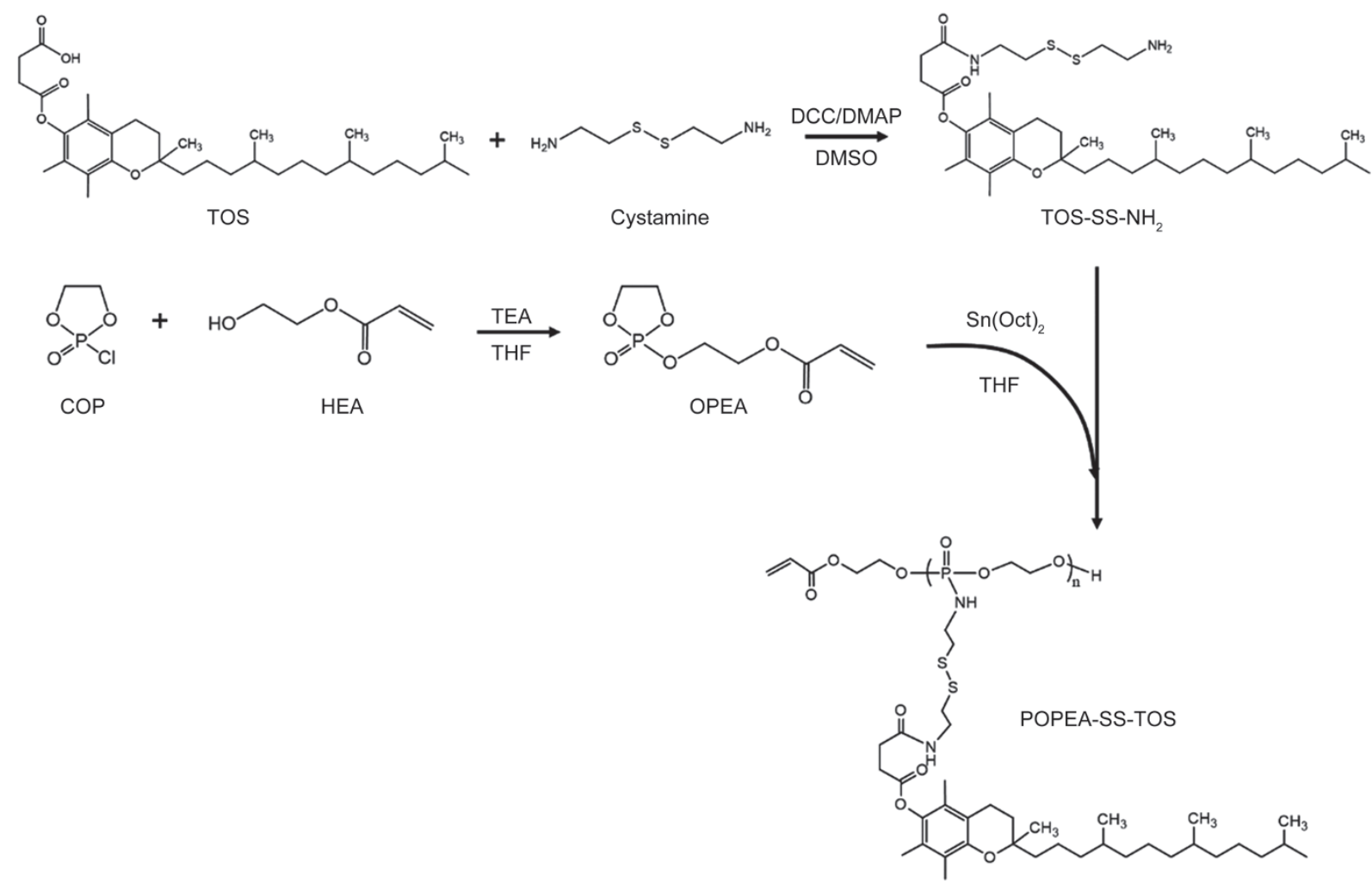

Scheme 1. Scheme of PSST copolymer synthesis.

pyrene verses the logarithm of the PSST copolymer concentration was plotted. The fluorescent ratio was small at low polymer concentrations but markedly increased at higher polymer concentrations (Figure 1C). Thus, the graph had two linear segments with different slopes, and the CMC value of the PSST copolymer was determined by the intersection point, which was at $0.0501 \mathrm{mg} / \mathrm{mL}$. This indicates that the micelles consisting of PSST copolymer possess an outstanding ability to resist dilution.

Biocompatibility is one of the most important properties of polymers used for cancer therapy. The in vitro cytotoxicity of the PSST copolymer against A2780 and A2780/PTX cells was evaluated using an MTT assay. After treatment for $48 \mathrm{~h}$, the cell viabilities for A2780 and A2780/PTX cells treated with PSST polymers at final concentrations ranging from 12.5 to 200 $\mu \mathrm{g} / \mathrm{mL}$ were all above $85 \%$ (Figure 1D). This result demonstrates that the PSST polymer is optimally biocompatible with A2780 and A2780/PTX cells. The low cytotoxicity of the PSST polymer is important for its safe use as a drug delivery vector.

\section{Multidrug resistance against PSST polymer}

It has been reported that the TOS moiety inhibits P-gp ${ }^{[45]}$. Herein, we tested whether the PSST polymer still induced an inhibitory effect on P-gp. The calcein-AM fluorescent assay was used to evaluate the inhibition of P-gp by PSST polymer. When calcein-AM passes through the cell membrane, the chemical is converted to fluorescent products by the esterase in the cells. However, P-gp can efflux calcein-AM out of cells, resulting in decreased production of fluorescence products in the cells. This process can be suppressed by the administration of P-gp inhibitors.

As shown in Figure 2A and 2B, weak fluorescence was detected in untreated A2780/PTX cells because of their strong efflux of calcein-AM. However, PSST polymer caused a higher retention of calcein-AM in A2780/PTX cells, indicating that it inhibits the efflux mediated by P-gp, similar to the positive control verapamil. In contrast, due to the low expression of P-gp in A2780 cells, both the untreated and treated A2780 cells showed strong green fluorescence (Figure 2C). Thus, A2780 cells served as a negative control for A2780/PTX cells in this experiment.

Furthermore, the inhibition of P-gp activity by PSST polymer in A2780/PTX cells was evaluated by Western blotting and FCM analyses. As shown in Figure 2D, the overexpression of P-gp in A2780/PTX cells was confirmed by comparing to A2780 cells. The P-gp expression in the A2780/PTX cells was significantly reduced by PSST polymer treatment. In addition, the inhibition of P-gp by PSST polymer was verified by FCM analysis (Figure 2E). As demonstrated in previous studies, TOS materials inhibited P-gp expression by reducing the ATPase activity of P-gp. However, this P-gp inhibition effect greatly depends on the amount of TOS moiety. Herein, these polymerized TOS moieties in PSST polymers could be released rapidly in response to the high concentration of GSH in cancer cells, following the fracture of disulfide bonds. Therefore, P-gp function could be significantly inhibited by 
A

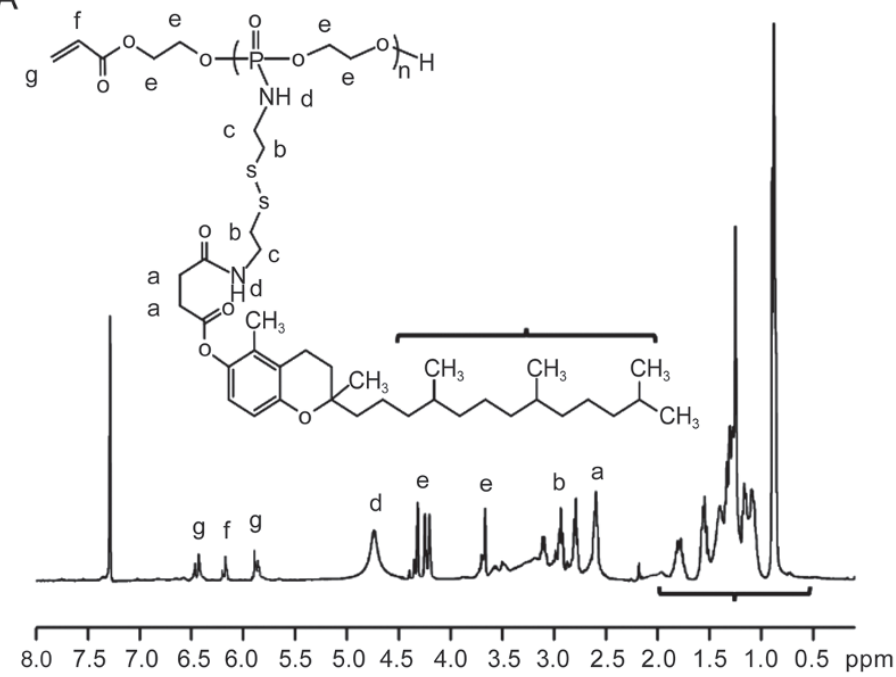

C

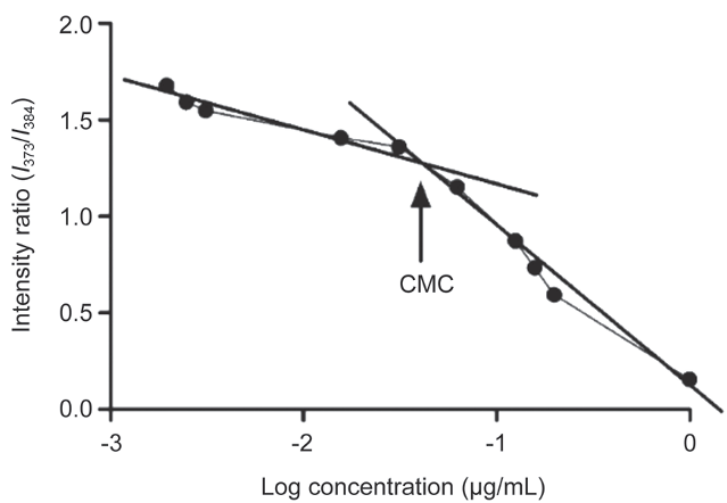

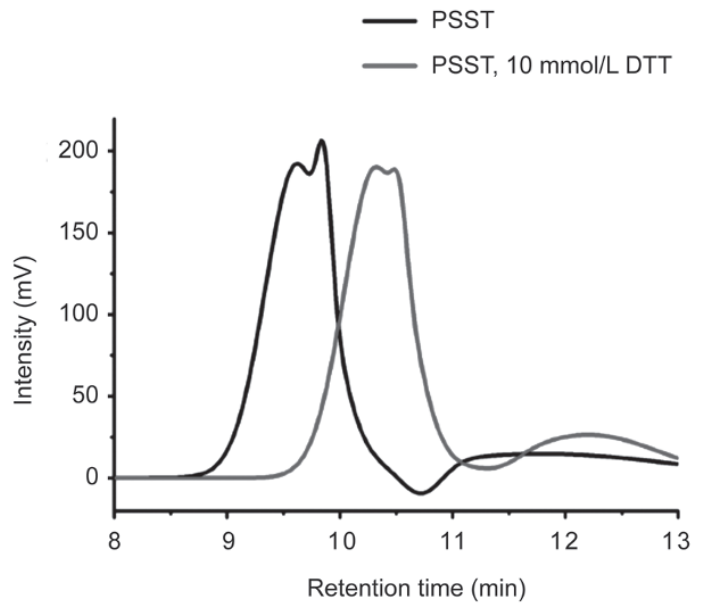

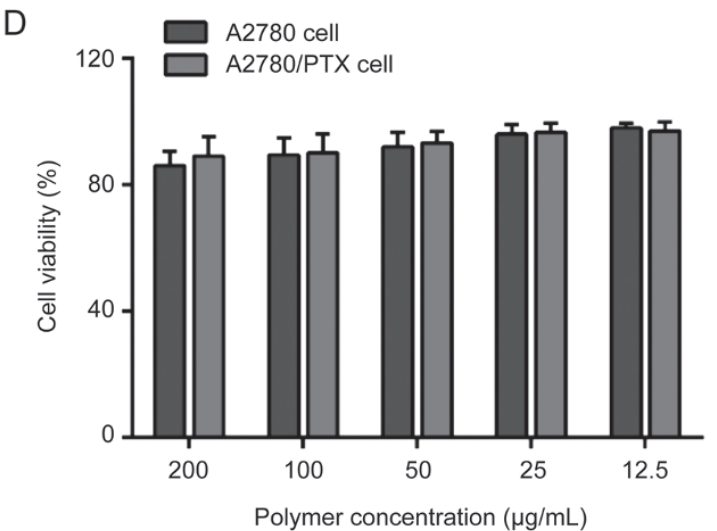

Figure 1. The chemical structure and ${ }^{1} \mathrm{H}$ NMR spectrum of PSST polymer are shown in (A). The gel permeation chromatography (GPC) curves of PSST polymer treated with or without $10 \mathrm{mmol} / \mathrm{L}$ DTT for $6 \mathrm{~h}$ were determined (B). The critical micellar concentration (CMC) value of PSST polymer was determined by the change of pyrene fluorescence (C). The MTT assay was used to examine viability of A2780 and A2780/PTX cells treated with PSST polymer for $48 \mathrm{~h}(\mathrm{D})$.

PSST polymer. In summary, these results indicate that the PSST polymer possesses a strong inhibitory effect against P-gp activity in A2780/PTX cells.

\section{Characterization of PTX/PSST-M}

With our encapsulation procedure, PTX was loaded into PSST micelles, with a LE and EE of $6.6 \% \pm 1.3 \%$ and $71.9 \% \pm 4.5 \%$, respectively. The particle size distribution and zeta potential of polymeric micelles are important parameters for applications in drug delivery. These parameters of our micelles are listed in Table 1. There was no significant difference in particle size distribution and zeta potential between empty PSST-M and PTX/PSST-M. The average size of the PTX/PSST-M was $68.1 \pm 4.9 \mathrm{~nm}$, which is within the optimal range to maintain a low level of reticuloendothelial system endocytosis, minimal renal excretion, and a strong enhanced permeability and

Table 1. Characterization of PSST micelles.

\begin{tabular}{lcccc}
\hline Samples & Particle size $(\mathrm{nm})$ & PDI & Zeta potential $(\mathrm{mV})$ & LE $(\%)$ \\
\hline Blank PSST-M & $60.7 \pm 5.8$ & $0.12 \pm 0.03$ & $-21.7 \pm 4.2$ & - \\
PTX/PSST-M & $68.1 \pm 4.9$ & $0.10 \pm 0.04$ & $-28.6 \pm 3.7$ & - \\
\hline
\end{tabular}

Note: PDI, polydispersity index; LE, loading efficiency; EE, encapsulation efficiency; PSST-M, poly(phosphate ester)-SS-D- $\alpha$-tocopherol succinate micelles; PTX/PSST-M, paclitaxel-loaded PSST micelles. 
A
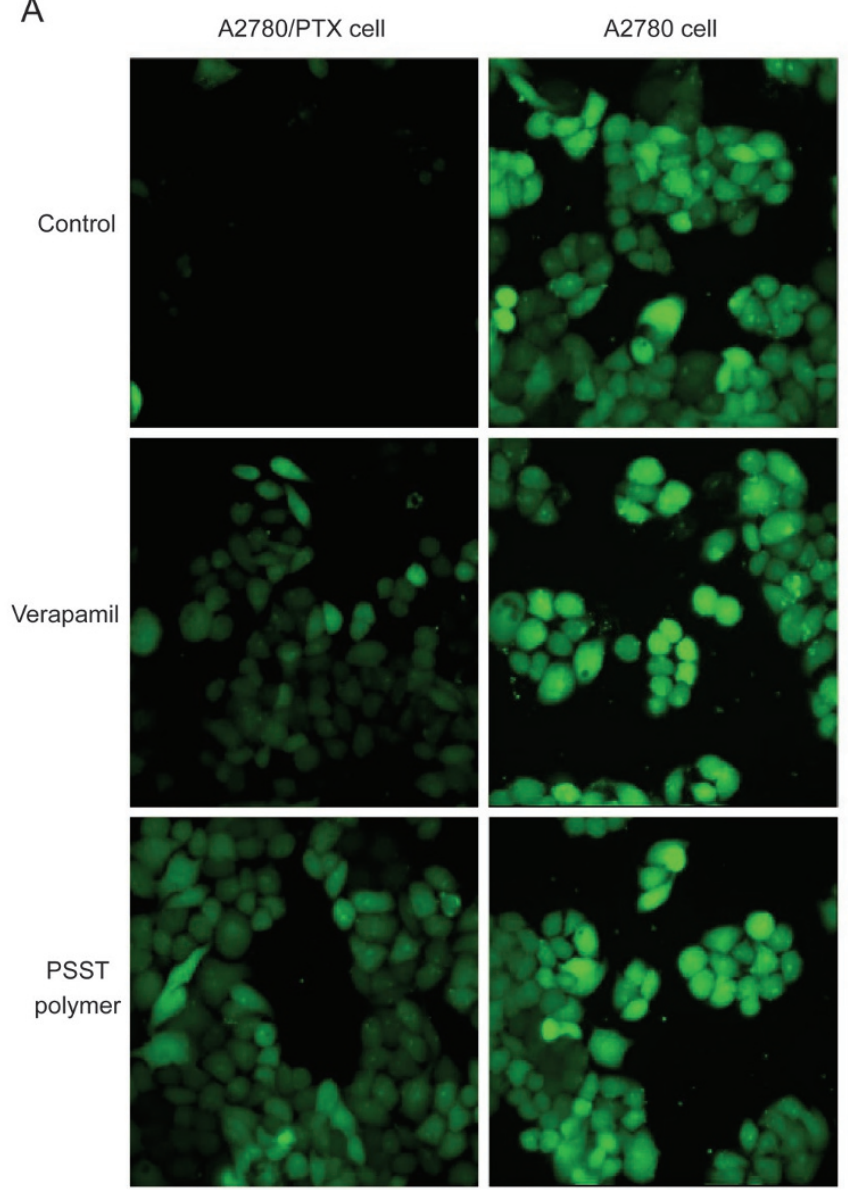

D
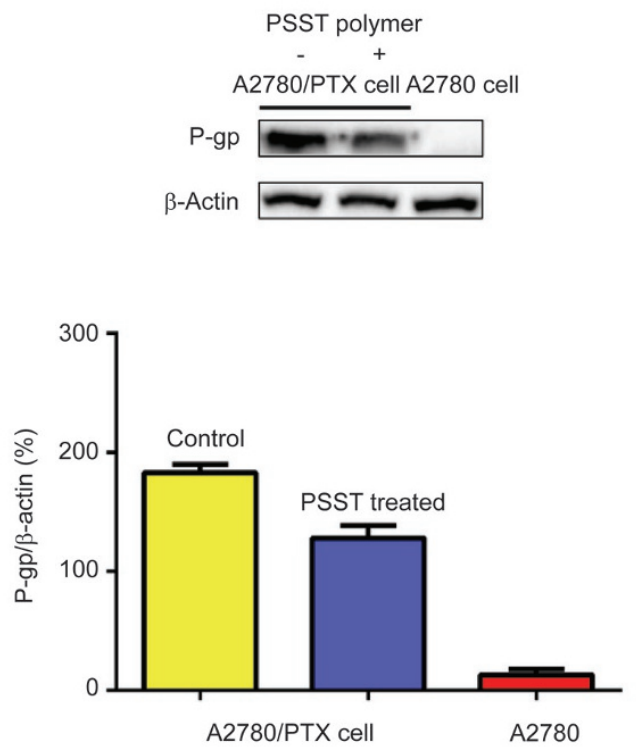

B
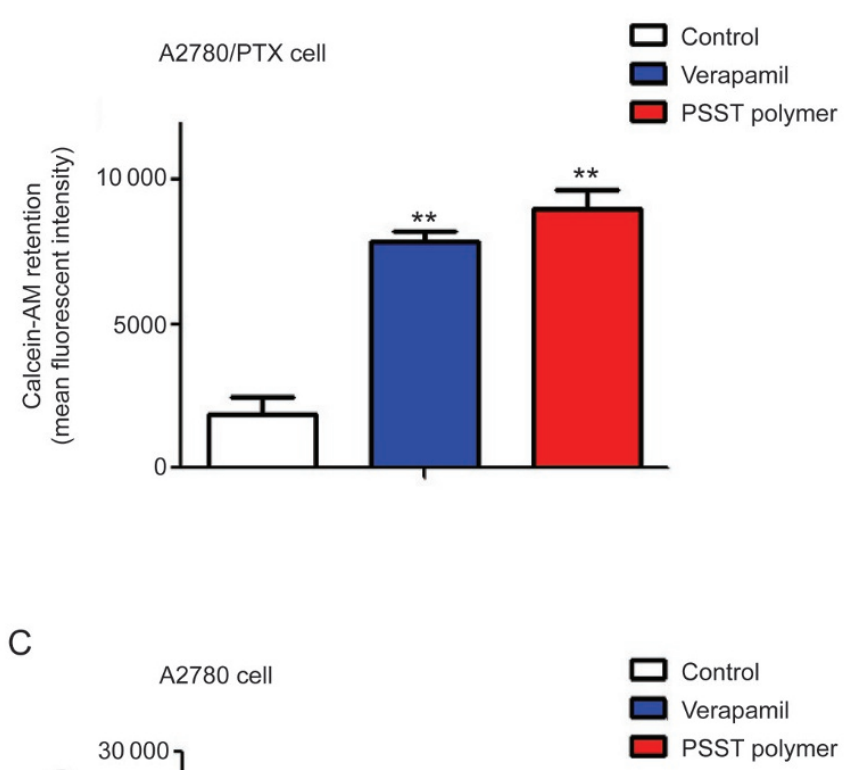

$\mathrm{E}$
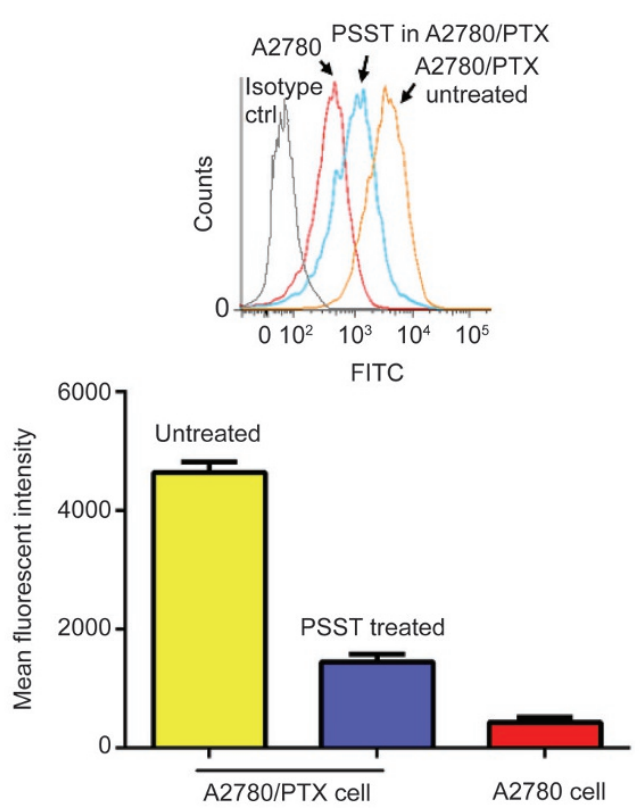

Figure 2. Images of cellular accumulation of calcein-AM in A2780/PTX and A2780 were taken after treated with vehicle (control), $0.1 \mu \mathrm{g} / \mathrm{mL}$ of verapamil (positive control), or $0.1 \mathrm{mg} / \mathrm{mL}$ of PSST polymer (A). The cellular accumulation of calcein-AM in A2780/PTX (B) and A2780 cells (C) was measured by FCM. The expression of P-gp in A2780/PTX cells treated with $0.1 \mathrm{mg} / \mathrm{mL}$ of PSST polymer was examined by Western blotting (D) and FCM analysis (E). ${ }^{*} P<0.05$ vs untreated A2780/PTX cells. 
retention (EPR) effect for passive tumor targeting ${ }^{[46,47]}$. The representative size distribution and a TEM image of the PTX/ PSST-M are shown in Figure $3 \mathrm{~A}$ and $3 \mathrm{~B}$, respectively. The TEM image in Figure 3B shows the homogeneous spherical structures of the PTX/PSST-M. The zeta potential is an important indicator for characterizing the state of the NP surface and predicting the long-term stability of NPs. The absolute value of zeta potential over $20 \mathrm{mV}$ for the PTX/PSST-M suggested that they remain stable for a long time (Figure 3C).

\section{Redox-sensitive property of the PSST micelles}

We observed a redox-responsive decrease in the molecular weight of the PSST polymer by GPC in the presence of 10 $\mathrm{mmol} / \mathrm{L}$ DTT. We next examined the redox-sensitive disassembly of the PSST micelles. Nile red (NR), a sensitive twisted intramolecular charge transfer probe, has been reported to exhibit strong fluorescence in a hydrophobic environment and weak fluorescence in aqueous medium ${ }^{[48]}$. NR was loaded into the PSST micelles via the same procedure used to make the PTX/PSST-M, and the changes in their fluorescence signals after exposure to $10 \mathrm{mmol} / \mathrm{L}$ DTT were recorded. Without DTT, the NR/PSST-M exhibited strong fluorescence due to the encapsulation of NR in the hydrophobic domain of the micellar core (Figure 3D). However, dramatic quenching of the fluorescence signal was observed in the NR/PSST-M exposed to $10 \mathrm{mmol} / \mathrm{L}$ DTT, which mimics the redox environment in cancer cells. This is due to increased dissociation of PSST micelles and the release of NR dye into the aqueous phase in response to DTT. This result suggests that the disassembly of PSST micelles is redox-responsive.

In addition, the changes in the size distribution of the PTX/ PSST-M in the presence of various concentrations of DTT for $24 \mathrm{~h}$ were monitored by DLS to detect reduced triggered disassembly $^{[22]}$. As shown in Figure 3E, after $24 \mathrm{~h}$ of exposure to $10 \mu \mathrm{mol} / \mathrm{L}$ DTT, which is equivalent to the typical GSH concentration in normal tissues, no marked change in size was detected over time. However, at $10 \mathrm{mmol} / \mathrm{L}$ DTT, which is equivalent to the GSH concentration found in tumor tissues, the mean particle size of the PTX/PSST-M significantly increased over time. This was due to the cleavage of the disulfide linkages in the micellar core. The particle size of the PTX/PSST-M exceeded $280 \mathrm{~nm}$ at $6 \mathrm{~h}$ and markedly increased to over $560 \mathrm{~nm}$ at $12 \mathrm{~h}$. This result indicates that the PTX/ PSST-M quickly disassembled in a high-redox environment, which is common in tumor cells, and maintained stable under normal conditions.

To further investigate the redox-sensitive destabilization of the hydrophobic micellar core, we evaluated PTX release from the PSST micelles at different concentrations of DTT. The release of PTX from the PTX/PSST-M was inefficient and slow in the presence of $10 \mu \mathrm{mol} / \mathrm{L}$ DTT, with less than $30 \%$ of total PTX released at $48 \mathrm{~h}$ (Figure 3F). The slow release behavior would lead to low intracellular PTX concentrations and low cytotoxicity to normal cells. In the medium with $10 \mathrm{mmol} / \mathrm{L}$ DTT, a markedly accelerated release of PTX from the PTX/ PSST-M was observed, which was similar to the release pro- file of free PTX. For instance, at $24 \mathrm{~h}$, compared to only $26.4 \%$ of PTX released from the PSST micelles in $10 \mu \mathrm{mol} / \mathrm{L}$ DTT, approximately $65.7 \%$ and $82.1 \%$ of PTX was released from the PSST micelles in $10 \mathrm{mmol} / \mathrm{L}$ DTT and from free PTX, respectively. Thus, the PTX/PSST-M achieve fast intracellular PTX release in high-redox environments and facilitate the ability of the loaded drug to overcome resistance.

\section{Intracellular accumulation of PTX/PSST-M}

The cellular uptake of free PTX and PTX/PSST-M containing $5 \mu \mathrm{mol} / \mathrm{L}$ PTX was quantitatively analyzed in A2780 and A2780/PTX cells. As shown in Figure 4A, increasing concentrations of free PTX were accumulated in A2780 cells in a timedependent manner, but much lower concentrations of PTX were accumulated in A2780/PTX cells after treatment for $4 \mathrm{~h}$, indicating strong drug efflux from A2780/PTX cells. However, the efflux of PTX/PSST-M was not apparent in A2780/ PTX cells with the over-expressed P-gp, as the uptake profile of PTX/PSST-M in A2780/PTX cells was similar to that of free PTX in A2780 cells (Figure 4B). After loaded in PSST-M, significantly enhanced rates and amounts of PTX uptake were observed in both A2780 and A2780/PTX cells. Compared to the cells exposed to free PTX, more than 6- and 28-fold PTX was accumulated in the A2780 and A2780/PTX cells, respectively, that were treated with PTX/PSST-M for $4 \mathrm{~h}$. In addition, during the $4 \mathrm{~h}$ treatment of the A2780/PTX cells, the efflux rate of free PTX $(0.65[\mathrm{PTX}](\mu \mathrm{g}) /[$ protein] $(\mathrm{mg}) / \mathrm{h})$ was significantly higher than that of the PTX/PSST-M (0.015 [PTX] $(\mu \mathrm{g}) /[$ protein $](\mathrm{mg}) / \mathrm{h})$. These results demonstrate that encapsulation of PTX in PSST micelles facilitates the ability of PTX to enter cells and to resist drug efflux.

As a substrate for the P-gp efflux pump, Rho-123 is often used as a fluorescent probe to visualize the accumulation behavior in resistant cancer cells. In the present study, Rho123 was loaded into PSST micelles and incubated with A2780/ PTX cells to investigate the effects of encapsulation in PSST micelles on the intracellular uptake efficiency and the P-gpmediated efflux of internalized drugs. By detecting the autofluorescence of Rho-123, FCM analysis revealed increased accumulation of Rho-123/PSST-M in the A2780/PTX cells in a time-dependent manner (Figure 4C). It was noted that exposure of the A2780/PTX cells to free Rho-123 did not lead to increased intracellular Rho-123 accumulation compared with untreated A2780/PTX cells. However, a significant retention of Rho-123 was observed in cells exposed to either the mixture of Rho-123 and PSST-M or the Rho-123/PSST-M. The 2.6-fold increase in intracellular Rho-123 accumulation in the A2780/ PTX cells exposed to the mixture of Rho- 123 and PSST-M for $4 \mathrm{~h}$ may be due to the inhibition of P-gp-mediated drug efflux by the PSST polymer. However, the Rho- 123 in the mixture may still be pumped out of the cells by the remaining P-gp activity. Rho-123/PSST-M treatment of the A2780/PTX cells resulted in 11.8- and 5.2-fold increases in intracellular Rho123 accumulation compared to treatment with free Rho- 123 and the Rho-123+PSST-M mixture, respectively, due to the synergistic effects of the nano-scale carrier and P-gp inhibition 
A

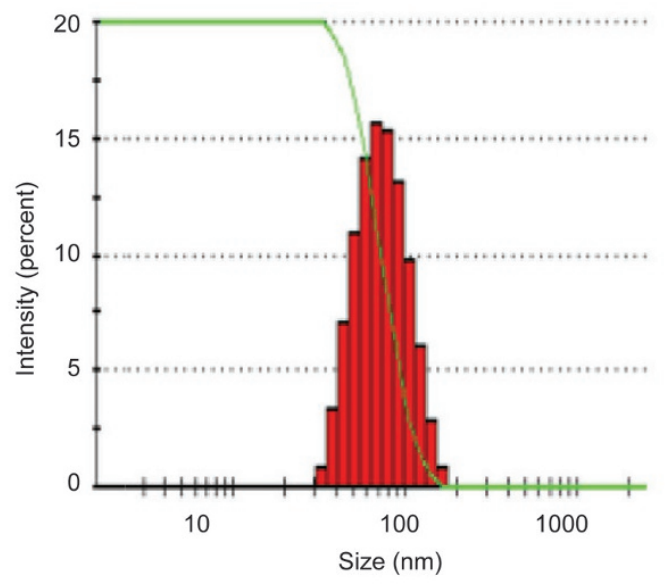

C

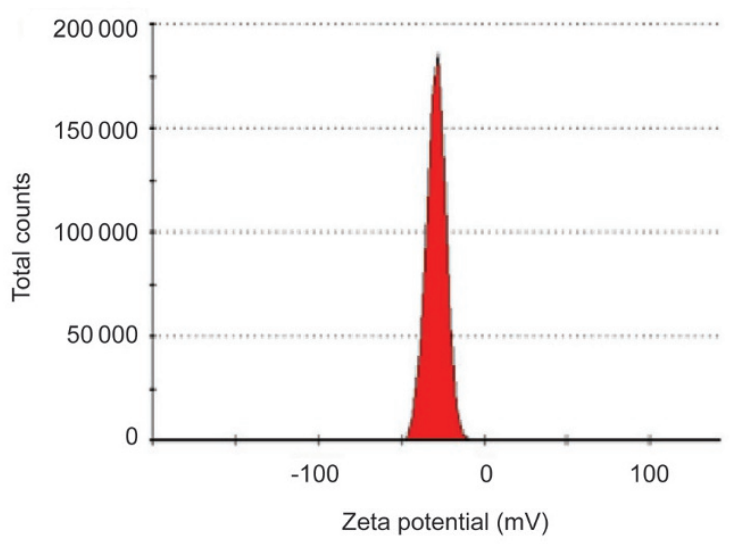

E

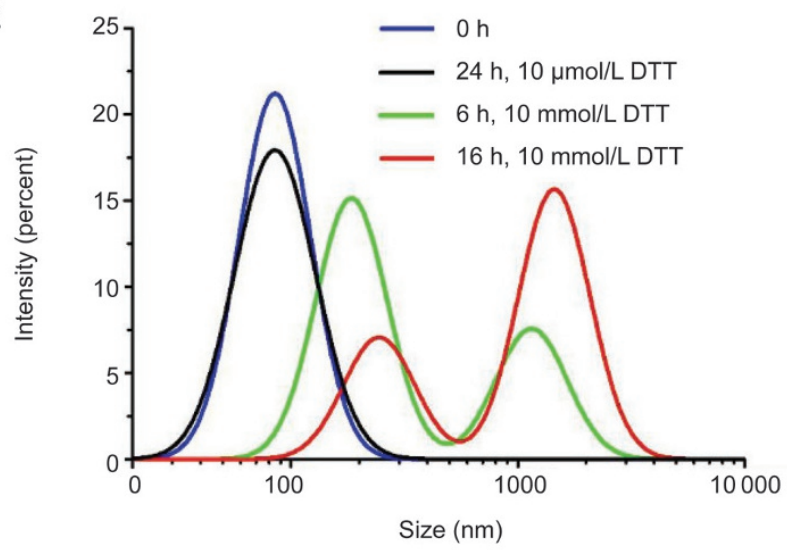

B
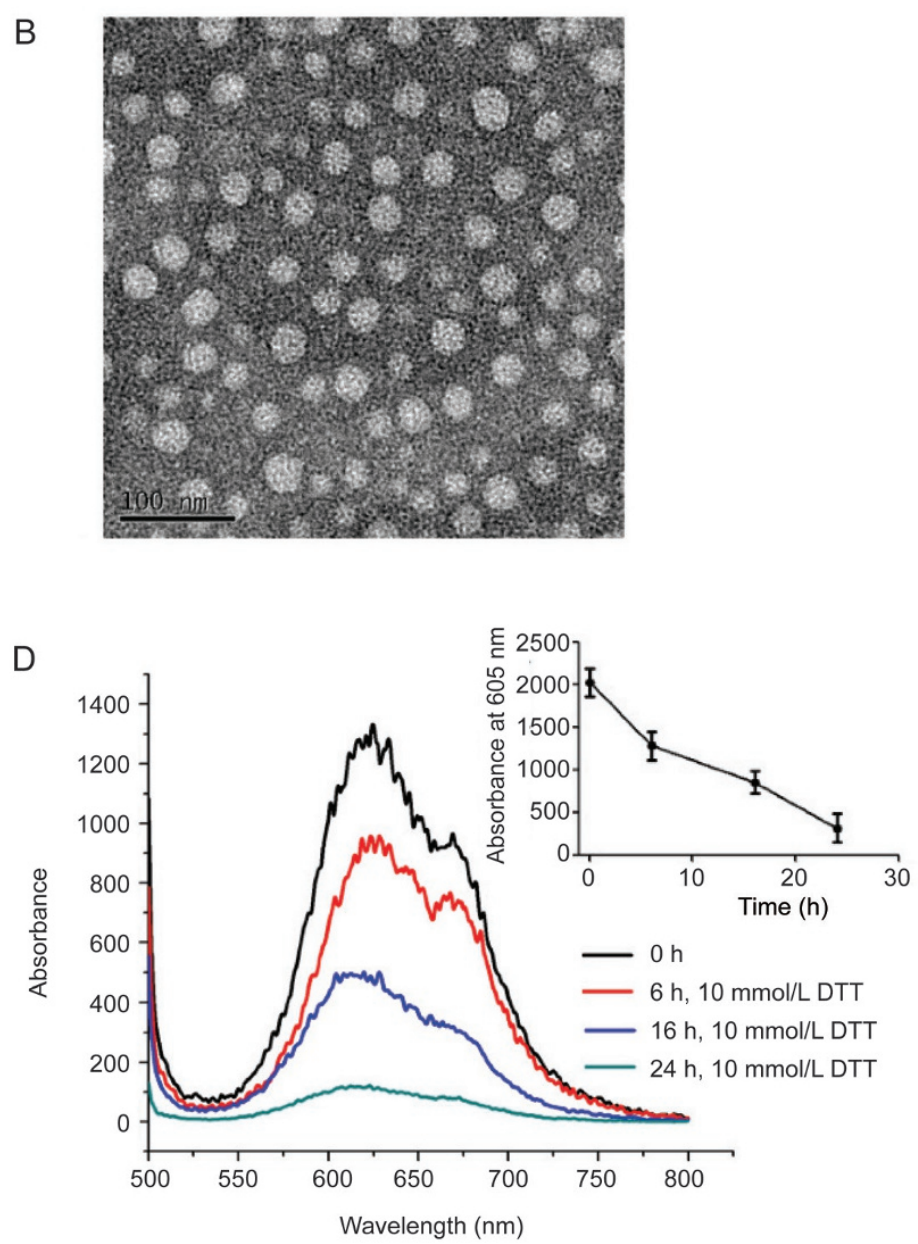

F

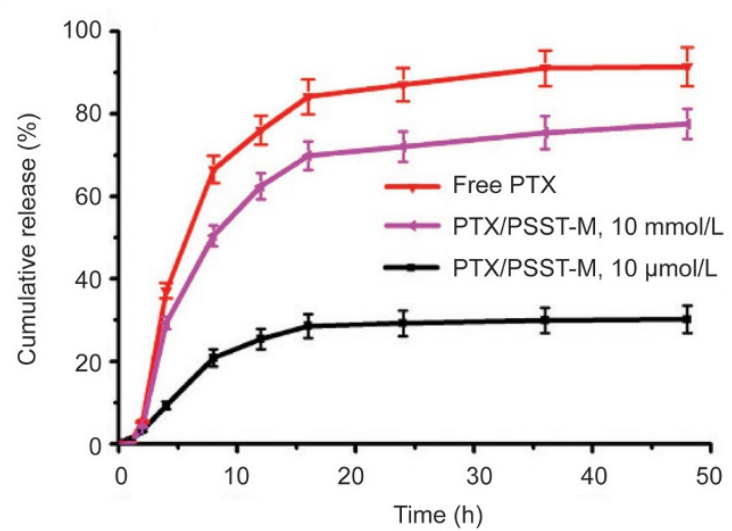

Figure 3. Characterization of PTX-loaded PSST micelles (PTX/PSST-M). The particle size distribution of PTX/PSST-M was measured by dynamic light scattering (DLS; A). A representative image of PTX/PSST-M was taken by a transmission electron microscopy (B). The scale bar represents $100 \mathrm{~nm}$. The zeta potential of PTX/PSST-M was measured by DLS (C). The fluorescence spectrum of Nile red-loaded PSST micelles in presence of 10 mmol/L DTT is shown in (D). The changes in size distribution of PTX/PSST-M in presence of $10 \mathrm{mmol} / \mathrm{L}$ DTT were examined by DLS (E). The in vitro release profiles of PTX from free PTX or PTX/PSST-M to the release medium with or without $10 \mathrm{mmol} / \mathrm{L}$ DTT were measured by dialysis (F).

properties of the PSST polymer. Therefore, stronger green Rho-123 fluorescence was observed in the A2780/PTX cells treated with Rho-123/PSST-M compared to the cells treated with free PTX or the Rho-123+PSST-M (Figure 4D).

Next, the uptake pathways of PSST-M were investigated by using various endocytotic inhibitors. As shown in Figure 
A

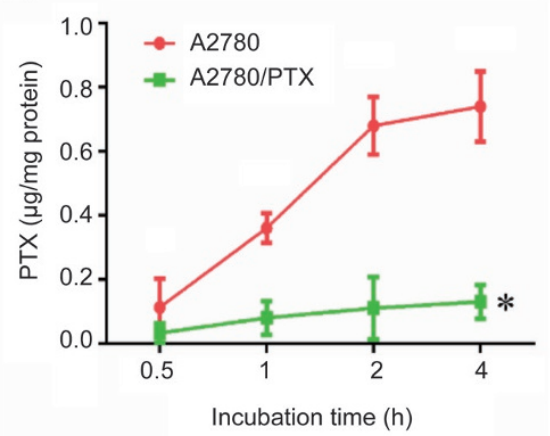

B

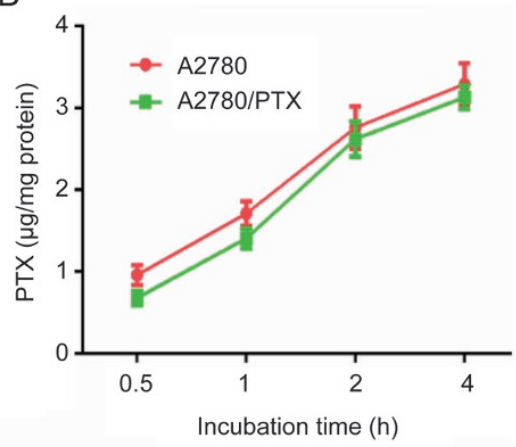

C

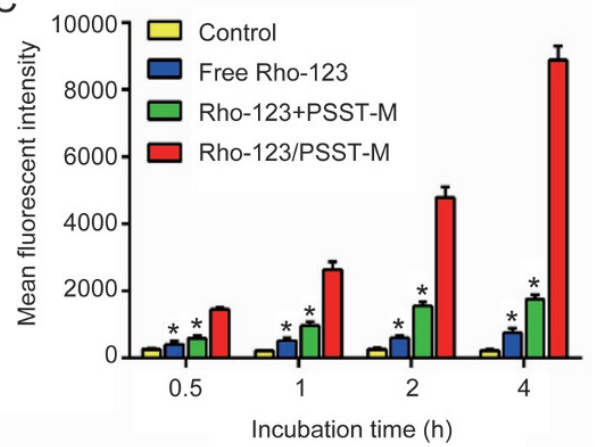

D

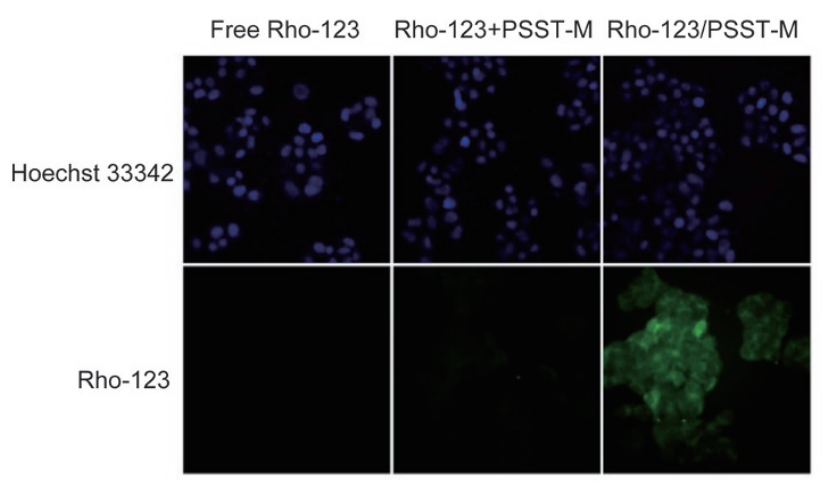

E

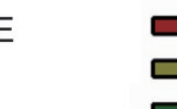

1: Hexamethylene amiloride 2: Methyl- $\beta$-cyclodextrin 3: Chlorpromazine
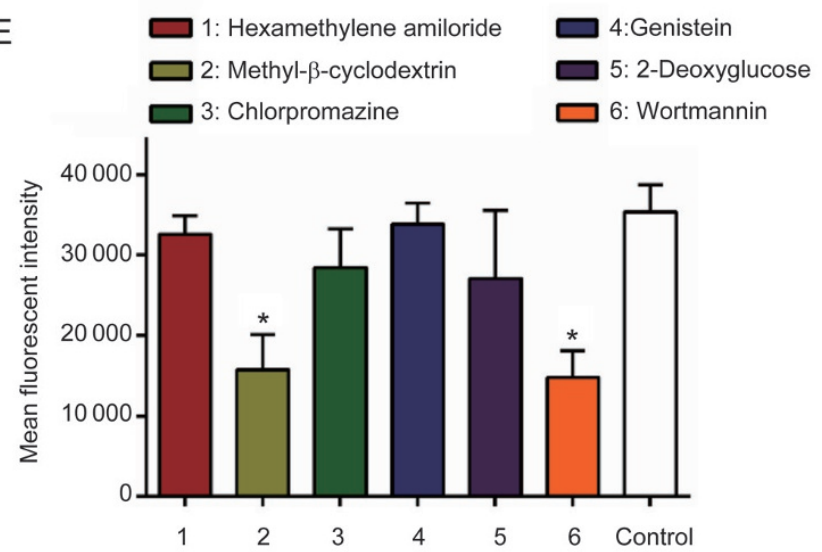

Figure 4. PTX accumulation was examined in A2780 and A2780/PTX cells after incubation with free PTX (A) and PTX/PSST-M (B) at an equivalent PTX concentration of $5 \mu \mathrm{mol} / \mathrm{L}\left({ }^{*} P<0.05\right.$ vs free PTX in A2780 cells). Rho-123/PSST-M accumulation in A2780/PTX cells was analyzed by a flow cytometer (C) and a fluorescence microscope (D) after treatment for $4 \mathrm{~h} \mathrm{(}{ }^{*} \mathrm{P}<0.05$ vs Rho-123/PSST-M). The uptake mechanisms of PSST micelles in A2780/PTX cells were investigated by pretreatment with various inhibitors against endocytosis $(E) ;{ }^{*} P<0.05$ vs control A2780/PTX cells without pretreatment of inhibitors.

4E, the cellular uptake of Rho-123/PSST-M in the A2780/ PTX cells was significantly reduced by pretreatment with wortmannin and methyl- $\beta$-cyclodextrin. Wortmannin has been demonstrated to be an inhibitor of micropinocytosis, which blocks phosphoinositide 3-kinase-related spontaneous surface ruffling ${ }^{[49]}$. Thus, the micropinocytosis pathway may be involved in the cellular internalization of PSST micelles. In addition, the 55\% reduction in the cellular uptake of PSST micelles by methyl- $\beta$-cyclodextrin indicated the involvement of caveolin-dependent endocytosis. In summary, the PTX/ PSST-M enhance the intracellular uptake and accumulation of PTX via the synergistic effects of the nanocarrier and the P-gp inhibition properties of the PSST polymer, and the micropinocytosis and caveolin-dependent endocytosis pathways may be involved in this process.

\section{In vitro cytotoxicity measured by the MTT assay}

In light of P-gp inhibition and the higher cellular uptake of PSST micelles, the ability of PTX/PSST-M to induce cytotoxicity in A2780 and A2780/PTX cells was examined. No significant cytotoxicity was detected in cells treated with blank PSST micelles containing $12.5-200 \mu \mathrm{g} / \mathrm{mL}$ PSST polymer (Supple- mentary Figure S1), indicating their high biocompatibility. Free PTX and the PTX+PSST-M displayed similar cytotoxicity against A2780 cells at $48 \mathrm{~h}$ and $72 \mathrm{~h}$ post-drug administration, which was dependent on concentration and time (Figure 5A and 5C). Compared to free PTX, the PTX/PSST-M exhibited higher cytotoxicity against A2780 cells due to its higher cellular accumulation. In A2780/PTX cells, the PTX+PSST-M exhibited much higher cytotoxicity than free PTX (Figure 5B and 5D). This may be due to inhibition of P-gp by PSST polymer and less PTX being pumped out of the cells. Notably, the PTX/PSST-M caused more cytotoxicity against A2780/PTX cells than either free PTX or PTX+PSST-M treatment at the equivalent PTX concentrations. To quantitatively compare the cytotoxicity among free PTX, PTX+PSST-M, and PTX/PSST-M, their $\mathrm{IC}_{50}$ values were calculated based on the cytotoxicity curves. When A2780/PTX cells were treated with free PTX, PTX+PSST-M, or PTX/PSST-M for $48 \mathrm{~h}$, the $\mathrm{IC}_{50}$ values of PTX were $61.51,29.99$ and $0.49 \mu \mathrm{mol} / \mathrm{L}$, respectively. After treatment for $72 \mathrm{~h}$, the $\mathrm{IC}_{50}$ values of PTX in free PTX, PTX+PSST-M or PTX/PSST-M were 43.27, 19.97, and $0.19 \mu \mathrm{mol} / \mathrm{L}$, respectively. Compared to free PTX, the $\mathrm{IC}_{50}$ values of PTX in the PTX/PSST-M were 125.5- and 227.7-fold lower after treatment 

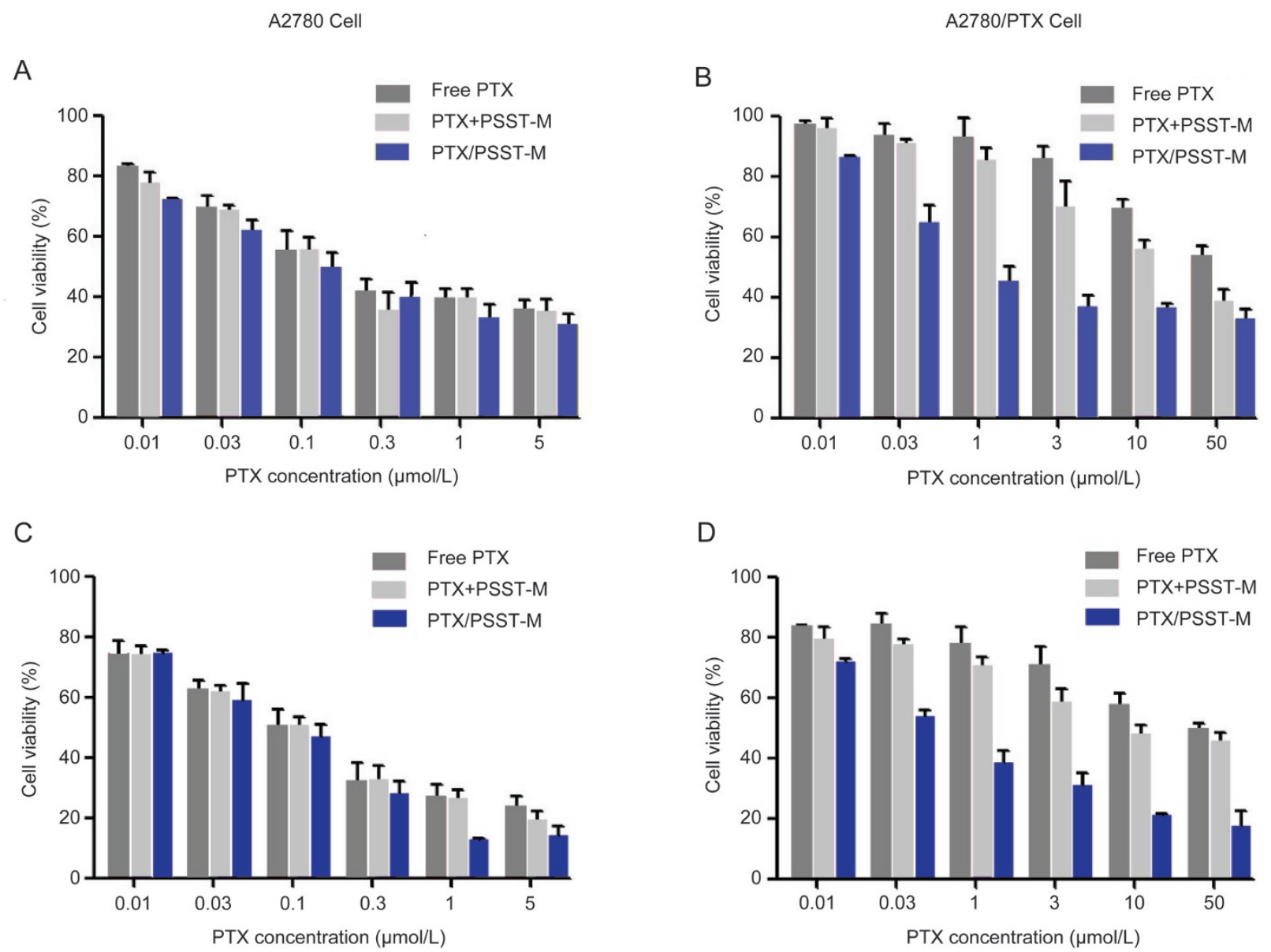

Figure 5. The MTT assay was used to evaluate cytotoxicity of free PTX, PTX+PSST-M, and PTX/PSST-M in A2780 and A2780/PTX cells after treatment for $48 \mathrm{~h}(\mathrm{~A}, \mathrm{~B})$ and $72 \mathrm{~h}(\mathrm{C}, \mathrm{D})$.

for $48 \mathrm{~h}$ and $72 \mathrm{~h}$, respectively. Therefore, the encapsulation of PTX in PSST micelles effectively sensitizes A2780/PTX cells to PTX treatment.

\section{Effects of PTX formulations on mitochondrial transmembrane potential, ATP levels, and ROS production}

The mitochondria transmembrane potential $\left(\Delta \psi_{\mathrm{m}}\right)$ is believed to be closely associated with the activity of mitochondria, and a decreased $\Delta \psi_{\mathrm{m}}$ is generally accepted as a hallmark of apoptosis ${ }^{[50]}$. Mitochondrial depolarization changes the fluorescence of JC-1 probes from red to green. Free PTX at 1 $\mu \mathrm{mol} / \mathrm{L}$ did not cause a significant change in red fluorescence in comparison to the untreated control (Figure 6A). However, at an equivalent PTX concentration of $1 \mu \mathrm{mol} / \mathrm{L}, \mathrm{PTX} / \mathrm{PSST}-\mathrm{M}$ induced a significant change in fluorescence in A2780/PTX cells, transforming red fluorescence to green fluorescence. The ratio of JC-1 red/green fluorescence was significantly decreased to $41.3 \% \pm 17.5 \%$ of the control ratio after treatment with the PTX/PSST-M (Figure 6B). However, after incubation with free PTX or the PTX+PSST-M, the JC-1 red/green values only decreased to $92.6 \% \pm 4.8 \%$ and $75.8 \% \pm 12.0 \%$ of the control ratio, respectively. These results indicate that the PTX/ PSST-M suppressed the mitochondrial transmembrane poten- tial and disrupt mitochondrial function.

Because mitochondrial activity is closely related to cellular energy metabolism, we investigated whether the PTX/PSST-M cause ATP depletion. Compared to untreated controls, we found that free PTX, PTX+PSST-M, and PTX/PSST-M induced significant decreases in intracellular ATP levels in A2780/PTX cells by $82.1 \% \pm 4.8 \%, 75.2 \% \pm 6.8 \%$, and $38.7 \% \pm 2.1 \%$, respectively (Figure 6C). P-gp exerts ATPase activity to hydrolyze ATP and provide energy for substrate efflux, and thus, ATP is essential for the efflux function of P-gp ${ }^{[51]}$. The PTX/PSST-M markedly decreased intracellular ATP levels, which may inhibit $\mathrm{P}$-gp-mediated drug resistance and prolong drug retention.

ROS promotes apoptosis in cancer cells, and thus, increasing ROS production may be an effective anticancer strategy. In this study, intracellular ROS production was examined by the DCFH-DA method. The data showed that neither free PTX nor the PTX+PSST-M resulted in significant increases in ROS levels in A2780/PTX cells compared to the untreated control (Figure 6D). However, the intracellular ROS level was significantly increased to $386.4 \% \pm 7.9 \%$ of control upon PTX/PSST-M treatment for $48 \mathrm{~h}$. 
A

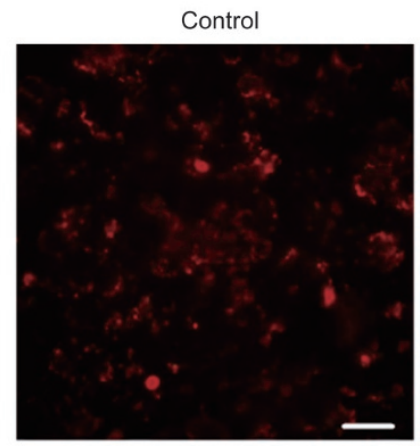

B

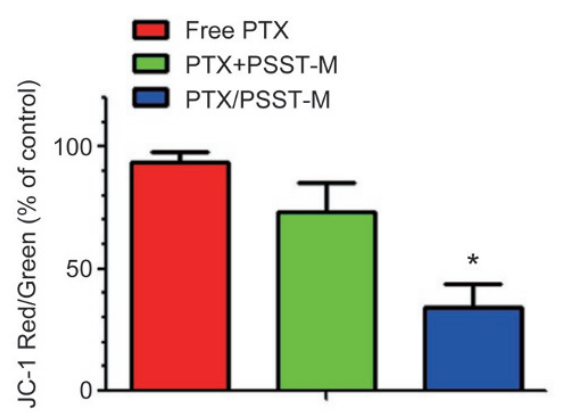

Free PTX

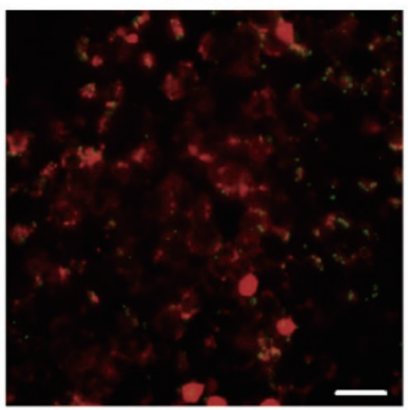

PTX+PSST-M

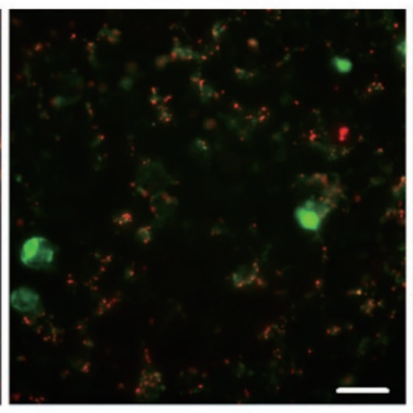

PTX/PSST-M

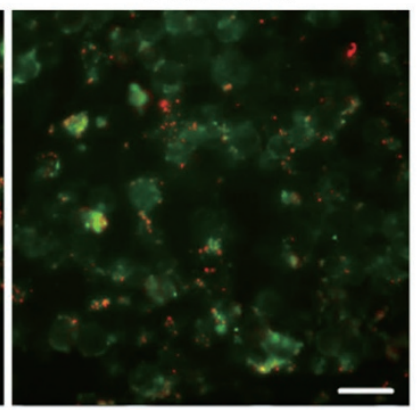

C

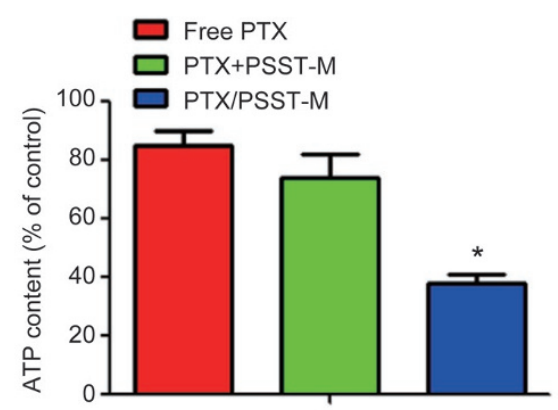

D

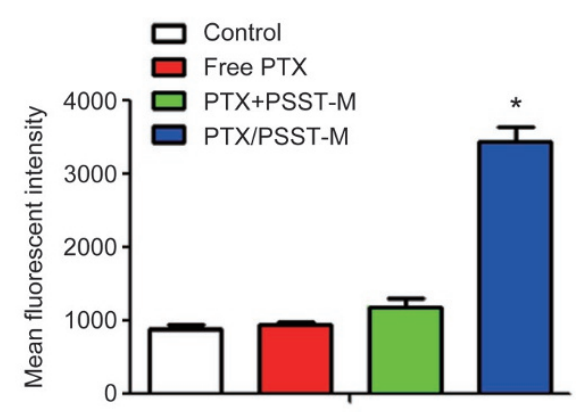

Figure 6. Mitochondrial transmembrane potential was measured in A2780/PTX cells treated with free PTX, PTX+PSST-M, or PTX/PSST-M at an equivalent PTX concentration of $1 \mu \mathrm{mol} / \mathrm{L}$ for $48 \mathrm{~h}(\mathrm{~A}, \mathrm{~B})$. Untreated cells acted as control. Following the same treatments, intracellular ATP levels (C) and production of reactive oxygen species (D) were measured. ${ }^{*} P<0.05$ vs the PTX+PSST-M group.

Effects of PTX formulations on cell cycle arrest, apoptosis, and caspase-3/7 activity

The cell cycle distribution was analyzed in A2780/PTX cells by FCM after treatment with various PTX formulations at an equivalent PTX concentration of $1 \mu \mathrm{mol} / \mathrm{L}$ for $48 \mathrm{~h}$ (Figure $7 \mathrm{~A})$. Compared to $9.34 \% \pm 0.74 \%$ of the untreated cells in the $\mathrm{G}_{2} / \mathrm{M}$ phase, free PTX, PTX+PSST-M, and PTX/PSST-M treatments resulted in $15.52 \% \pm 2.35 \%, 21.37 \% \pm 4.95 \%$, and $82.45 \% \pm 8.62 \%$ of cells in the $\mathrm{G}_{2} / \mathrm{M}$ phase, respectively (Figure 7C). Therefore, PTX/PSST-M induced the strongest effect on cell cycle arrest in A2780/PTX cells among all of the PTX formulations.

To explore whether the anticancer activity against A2780/ PTX cells induced by the various PTX formulations is due to the induction of cell apoptosis, we detected the apoptotic rate of A2780/PTX cells after treatment with various PTX formulations at an equivalent PTX concentration of $1 \mu \mathrm{mol} / \mathrm{L}$ for $48 \mathrm{~h}$. FITC-Annexin V assays detect early apoptosis by incorporating phosphatidyl serine into the external cytomembrane of apoptotic cells with a high affinity, and PI labels cell necrosis by entering cells through the damaged membrane. After treatment for $48 \mathrm{~h}$, the cell apoptotic rates were $12.1 \% \pm 2.8 \%$, $22.6 \% \pm 5.9 \%$, and $68.1 \% \pm 12.4 \%$ for the free PTX, PTX+PSST-M, and PTX/PSST-M groups, respectively (Figure 7B and 7D). These results demonstrate that PTX/PSST-M has a potent proapoptotic effect on A2780/PTX cells.

The activity of caspase $3 / 7$ is an important indicator of apoptosis and was determined in A2780/PTX cells treated with various PTX formulations at an equivalent PTX concentration of $1 \mu \mathrm{mol} / \mathrm{L}$ for $48 \mathrm{~h}$. The caspase $3 / 7$ activities in the A2780/PTX cells treated with free PTX and PTX+PSST-M were 2.1 \pm 1.2 - and 5.4 \pm 2.3 -fold higher than that in the untreated control (Figure 7E), which may be caused by PTX-induced cell cycle arrest at the $\mathrm{G}_{2} / \mathrm{M}$ phase. PTX/PSST-M treatment induced the strongest activation of caspase $3 / 7$, which was 23.5- and 4.3-fold higher than the activation of caspase 3/7 in the control and PTX+PSST-M groups, respectively. As activation of caspase $3 / 7$ is considered as the ultimate effector of apoptosis, these results indicate that the anti-cancer activity of the PTX/PSST-M in A2780/PTX cells is related to the activation of apoptotic pathways.

\section{Discussion}

Due to the severe obstacle of MDR in anticancer chemotherapy, various approaches have been implemented to surmount the overexpression of MDR-related ATP-binding cassette transporters, including P-gp, MRP1, and other resistance proteins. The most routine method is to co-deliver small molecule P-gp inhibitors such as verapamil with anticancer drugs ${ }^{[52]}$. However, such methods have not yet yielded promising outcomes because the high dosage of verapamil as a P-gp inhibitor far exceeds that as an antihypertension drug, possibly resulting in side-effects ${ }^{[53]}$. Additionally, the uniform pharmacokinetic profiles of P-gp inhibitors and anticancer agents 
A

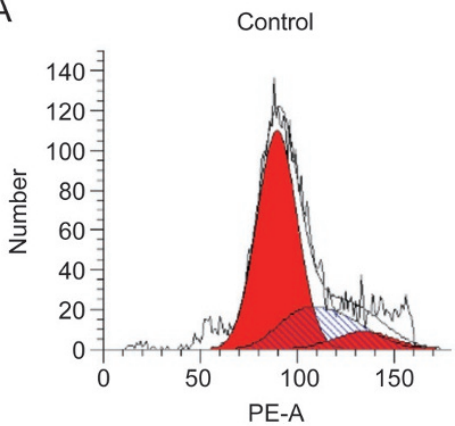

B

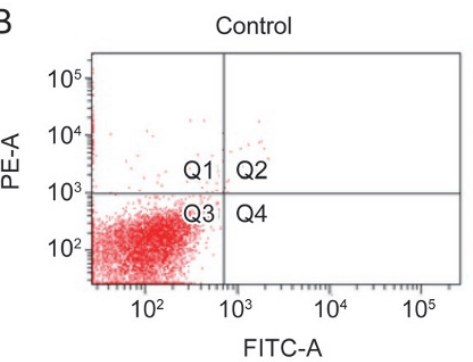

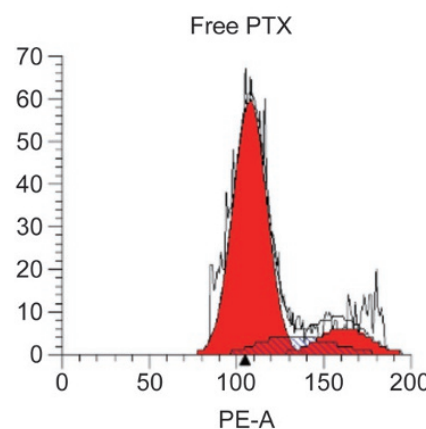

PE-A
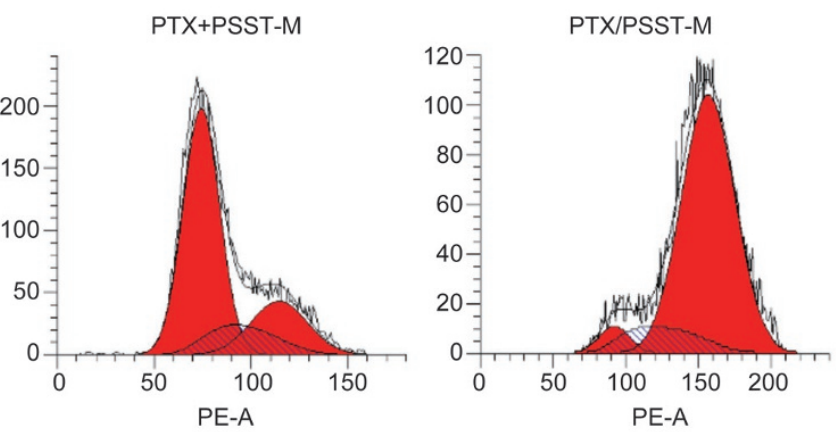
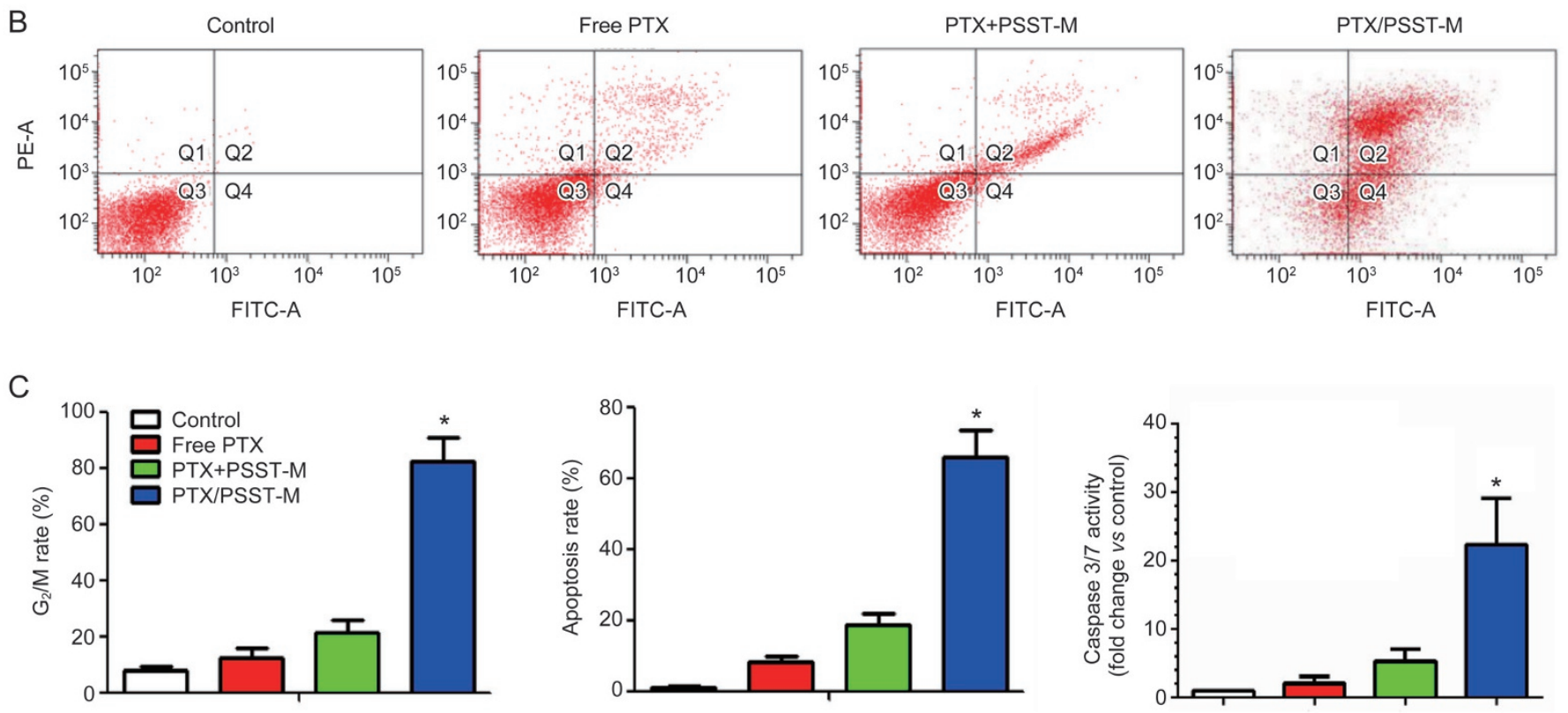

Figure 7. The cell cycle distribution (A) and apoptosis (B) in A2780/PTX cells were measured by a flow cytometer after treatment with various PTX formulations an equivalent PTX concentration of $1 \mu \mathrm{mol} / \mathrm{L}$ for $48 \mathrm{~h}$. In panel B, the top left quadrant (Q1) shows necrotic cells (Annexin V-FITC $\mathrm{PI}^{+}$), the top right quadrant (Q2) shows late apoptotic cells (Annexin $\mathrm{V}-\mathrm{FITC}^{+} \mathrm{PI}^{+}$), the bottom left quadrant (Q3) shows live cells (Annexin $\mathrm{V}^{-} \mathrm{FITC}^{-} \mathrm{PI}^{-}$), and the bottom right quadrant (Q4) shows early apoptotic cells (Annexin $\mathrm{V}-\mathrm{FITC}^{+} \mathrm{PI}^{-}$). The cell cycle distribution and arrest at $\mathrm{G}_{2} / \mathrm{M}$ phase were quantified (C). The total apoptotic rate was calculated as the sum of early apoptotic rate and late apoptotic rate (D). The caspase 3/7 activity in A2780/PTX cells was measured using the Caspase-glo $3 / 7$ Assay kit (E). ${ }^{*} P<0.05$ vs the PTX+PSST-M group.

also lead to undesirable combination efficiency. Thus, herein we focused on the development of biodegradable nanomaterials, ie, PSST polymer, to modulate P-gp and to deliver PTX via shell-core nanostructured micelles. In addition to improving the solubility of PTX, PSST polymers also increased the cellular accumulation of PTX by nanoscale carriers in combination of the P-gp suppression. Moreover, stimuli-responsive nano drug delivery systems have been designed to facilitate the escape of endosome/lysosomes to rapidly and thoroughly release cargo drugs into the cytoplasm after internalization ${ }^{[54]}$. The existence of redox potential differences in cytosolic GSH concentrations between tumor cells and normal cells provides the capability of fast disassembly of micelles. Therefore, the present study combined the advantages of micelles assembled from PSST polymers on P-gp inhibition and redox-responsive drug release.

In the present study, we compared the MDR reversal and anticancer efficiency of free PTX, a physical mixture of PTX and blank PSST micelles and PTX-loaded PSST micelles to determine the synergistic effect of MDR suppression by polymeric materials and nano-scaled vehicles with a shellcore structure. In cellular uptake experiments (Figure 4), the intracellular entry of the fluorescent P-gp substrate, Rho-123 into drug-resistance A2780 cells was significantly enhanced by incorporating the PSST polymer because of its P-gp suppression activity, as shown in Figure 2. However, because of the internalization advantage of nano-vehicles, much higher concentrations of Rho-123 loaded into the PSST micelles were able to accumulate in A2780/PTX cells compared to treatment with the physical mixture. The higher uptake process was subsequently investigated in relation to micropinocytosis and caveolin-dependent endocytosis pathways. As expected, the mixture of the PSST polymer resulted in the higher cellular uptake of PTX and followed the enhanced anti-proliferation 
and cell cycle arrest effect. Furthermore, based on the rapid drug release profile of PSST micelles in response to high GSH concentrations, the PTX loaded in the PSST micelles induced improved cytotoxicity effects and related mechanisms (Figure 5-7). Thus, these results demonstrated the value of the encapsulation of PTX in PSST micelles for overcoming MDR.

In summary, PSST polymers, $i e$, the amphiphilic a-tocopheryl succinate-based polyphosphoester conjugates containing disulfide linkages, were herein successfully synthesized to overcome MDR in drug-resistant human ovarian carcinoma. PTX was encapsulated into PSST micelles, which possessed remarkable redox-responsive drug release properties. Due to their advantages of nano-scale size and inhibitory effects on P-gp efflux by the a-TOS moiety, the PTX/PSST-M induced enhanced intracellular drug accumulation possibly via the micropinocytosis and caveolin-dependent endocytosis pathway. The PTX/PSST-M also reduced MDR in the PTX-resistant human ovarian cancer A2780/PTX cell line. Moreover, the PTX/ PSST micelles exhibited synergistic therapeutic mechanisms by increasing the mitochondria transmembrane potential and intracellular ROS levels, leading to activation of intrinsic apoptotic pathways and cell death. Collectively, the nano-sized PSST micellar vehicles, characterized by P-gp inhibitory functions and redox-responsive drug release, can be an effective drug delivery vector to overcome MDR.

\section{Acknowledgements}

This study was supported by the Macao Science and Technology Development Fund (096/2015/A3), the Research Fund of the University of Macau (MYRG2014-00033-ICMS-QRCM, MYRG2014-00051-ICMS-QRCM, MYRG2015-00171-ICMSQRCM and MYRG2016-00130-ICMS-QRCM), and the National Natural Science Foundation of China (81403120).

\section{Author contribution}

Feng-qian CHEN and Jin-ming ZHANG performed the experiments, analyzed the data, and drafted the manuscript; Xie-fan FANG, Yu-ling LIU, Hui LI, and Hua YU revised the manuscript; Yi-tao WANG and Mei-wan CHEN coordinated the experiment and revised and confirmed the paper.

\section{Supplementary information}

Supplementary information is available on the website of Acta Pharmacologica Sinica.

\section{References}

1 Vasey PA, Jayson GC, Gordon A, Gabra H, Coleman R, Atkinson R, et al. Phase III randomized trial of docetaxel-carboplatin versus paclitaxelcarboplatin as first-line chemotherapy for ovarian carcinoma. J Natl Cancer Inst 2004; 96: 1682-91.

2 Amiri-Kordestani L, Basseville A, Kurdziel K, Fojo AT, Bates SE. Targeting MDR in breast and lung cancer: discriminating its potential importance from the failure of drug resistance reversal studies. Drug Resist Updat 2012; 15: 50-61.

3 Huang IP, Sun SP, Cheng SH, Lee CH, Wu CY, Yang CS, et al. Enhanced chemotherapy of cancer using $\mathrm{pH}$-sensitive mesoporous silica nanoparticles to antagonize P-glycoprotein-mediated drug resistance. Mol
Cancer Ther 2011; 10: 761-9.

4 Yusuf RZ, Duan Z, Lamendola DE, Penson RT, Seiden MV. Paclitaxel resistance: molecular mechanisms and pharmacologic manipulation. Curr Cancer Drug Targets 2003; 3: 1-19.

5 Gao L, Liu G, Kang J, Niu M, Wang Z, Wang H, et al. Paclitaxel nanosuspensions coated with P-gp inhibitory surfactants: I. Acute toxicity and pharmacokinetics studies. Colloids Surf B Biointerfaces 2013; 111: $277-81$.

6 Kamazawa S, Kigawa J, Kanamori Y, Itamochi H, Sato S, Iba T, et al. Multidrug resistance gene-1 is a useful predictor of Paclitaxel-based chemotherapy for patients with ovarian cancer. Gynecol Oncol 2002; 86: 171-6.

7 Talekar M, Ouyang Q, Goldberg MS, Amiji MM. Co-silencing of PKM-2 and MDR-1 sensitizes multidrug resistant ovarian cancer cells to paclitaxel in a murine model of ovarian cancer. Mol Cancer Ther 2015; 14: 1521-31.

8 Szakacs G, Paterson JK, Ludwig JA, Booth-Genthe C, Gottesman MM. Targeting multidrug resistance in cancer. Nat Rev Drug Discov 2006; 5: 219-34.

9 Thomas H, Coley HM. Overcoming multidrug resistance in cancer: an update on the clinical strategy of inhibiting P-glycoprotein. Cancer Control 2003; 10: 159.

10 Pusztai L, Wagner P, Ibrahim N, Rivera E, Theriault R, Booser D, et al. Phase II study of tariquidar, a selective P-glycoprotein inhibitor, in patients with chemotherapy-resistant, advanced breast carcinoma. Cancer 2005; 104: 682-91.

11 Collnot EM, Baldes C, Wempe MF, Kappl R, Hüttermann J, Hyatt JA, et al. Mechanism of inhibition of P-glycoprotein mediated efflux by vitamin E TPGS: influence on ATPase activity and membrane fluidity. Mol Pharm 2007; 4: 465-74.

12 Collnot EM, Baldes C, Schaefer UF, Edgar KJ, Wempe MF, Lehr CM. Vitamin E TPGS P-glycoprotein inhibition mechanism: influence on conformational flexibility, intracellular ATP levels, and role of time and site of access. Mol Pharm 2010; 7: 642-51.

13 Duhem N, Danhier F, Préat V. Vitamin E-based nanomedicines for anti-cancer drug delivery. J Control Release 2014; 182: 33-44.

14 Wang J, Sun J, Chen Q, Gao Y, Li L, Li H, et al. Star-shape copolymer of lysine-linked di-tocopherol polyethylene glycol 2000 succinate for doxorubicin delivery with reversal of multidrug resistance. Biomaterials 2012; 33: 6877-88.

15 Tang LY, Wang YC, Li Y, Du JZ, Wang J. Shell-detachable micelles based on disulfide-linked block copolymer as potential carrier for intracellular drug delivery. Bioconjug Chem 2009; 20: 1095-9.

16 Sun TM, Du JZ, Yan LF, Mao HQ, Wang J. Self-assembled biodegradable micellar nanoparticles of amphiphilic and cationic block copolymer for siRNA delivery. Biomaterials 2008; 29: 4348-55.

17 Wang YC, Wang F, Sun TM, Wang J. Redox-responsive nanoparticles from the single disulfide bond-bridged block copolymer as drug carriers for overcoming multidrug resistance in cancer cells. Bioconjug Chem 2011; 22: 1939-45.

18 Shao H, Zhang M, He J, Ni P. Synthesis and characterization of amphiphilic poly ( $\varepsilon$-caprolactone)-b-polyphosphoester diblock copolymers bearing multifunctional pendant groups. Polymer 2012; 53 : 2854-63.

19 Cheng R, Meng F, Deng C, Klok HA, Zhong Z. Dual and multi-stimuli responsive polymeric nanoparticles for programmed site-specific drug delivery. Biomaterials 2013; 34: 3647-57.

20 Mura S, Nicolas J, Couvreur P. Stimuli-responsive nanocarriers for drug delivery. Nat Mater 2013; 12: 991-1003.

21 Yin Q, Shen J, Zhang Z, Yu H, Li Y. Reversal of multidrug resistance by stimuli-responsive drug delivery systems for therapy of tumor. Adv 
Drug Deliv Rev 2013; 65: 1699-715.

22 Li J, Huo M, Wang J, Zhou J, Mohammad JM, Zhang Y, et al. Redoxsensitive micelles self-assembled from amphiphilic hyaluronic aciddeoxycholic acid conjugates for targeted intracellular delivery of paclitaxel. Biomaterials 2012; 33: 2310-20.

23 Chen W, Zhong P, Meng F, Cheng R, Deng C, Feijen J, et al. Redox and $\mathrm{pH}$-responsive degradable micelles for dually activated intracellular anticancer drug release. J Control Release 2013; 169: 171-9.

24 Zhang Q, Vakili MR, Li XF, Lavasanifar A, Le XC. Polymeric micelles for GSH-triggered delivery of arsenic species to cancer cells. Biomaterials 2014; 35: 7088-100.

25 Yu ZQ, Yan JJ, You YZ, Zhou QH. Bioreducible and acid-labile poly (amido amine) s for efficient gene delivery. Int J Nanomedicine 2012; 7: 5819-32.

26 Guo Q, Luo P, Luo Y, Du F, Lu W, Liu S, et al. Fabrication of biodegradable micelles with sheddable poly (ethylene glycol) shells as the carrier of 7-ethyl-10-hydroxy-camptothecin. Colloids Surf B Biointerfaces 2012; 100: 138-45.

27 Steinbach T, Alexandrino EM, Wurm FR. Unsaturated poly(phosphoester)s via ring-opening metathesis polymerization. Polym Chem 2013; 4: 3800-6.

28 Zhang P, Hu L, Wang Y, Wang J, Feng L, Li Y. Poly ( $\varepsilon$-caprolactone)block-poly (ethyl ethylene phosphate) micelles for brain-targeting drug delivery: in vitro and in vivo valuation. Pharm Res 2010; 27: 2657 69.

29 Wang YC, Yuan YY, Du JZ, Yang XZ, Wang J. Recent progress in polyphosphoesters: from controlled synthesis to biomedical applications. Macromol Biosci 2009; 9: 1154-64.

30 Zhang J, Chen R, Fang X, Chen F, Wang Y, Chen M. Nucleolin targeting AS1411 aptamer modified $\mathrm{pH}$-sensitive micelles for enhanced delivery and antitumor efficacy of paclitaxel. Nano Res 2015; 8: 201-18.

31 Zhang Z, Tan S, Feng SS. Vitamin E TPGS as a molecular biomaterial for drug delivery. Biomaterials 2012; 33: 4889-906.

$32 \mathrm{Yu} \mathrm{H}, \mathrm{Xu} \mathrm{Z}$, Wang D, Chen X, Zhang Z, Yin Q, et al. Intracellular pHactivated PEG-b-PDPA wormlike micelles for hydrophobic drug delivery. Polym Chem 2013; 4: 5052-5.

33 Yu P, Yu H, Guo C, Cui Z, Chen X, Yin Q, et al. Reversal of doxorubicin resistance in breast cancer by mitochondria-targeted $\mathrm{pH}$-responsive micelles. Acta Biomater 2015; 14: 115-24.

34 Stuart MC, van de Pas JC, Engberts J. The use of Nile Red to monitor the aggregation behavior in ternary surfactant-water-organic solvent systems. J Phys Org Chem 2005; 18: 929-34.

35 Wang J, Yang G, Guo X, Tang Z, Zhong Z, Zhou S. Redox-responsive polyanhydride micelles for cancer therapy. Biomaterials 2014; 35 : 3080-90.

36 O'Connor R, Ooi MG, Meiller J, Jakubikova J, Klippel S, Delmore J, et al. The interaction of bortezomib with multidrug transporters: implications for therapeutic applications in advanced multiple myeloma and other neoplasias. Cancer Chemother Pharmacol 2013; 71: 1357-68.

37 Fung KL, Pan J, Ohnuma S, Lund PE, Pixley JN, Kimchi-Sarfaty C, et al. MDR1 synonymous polymorphisms alter transporter specificity and protein stability in a stable epithelial monolayer. Cancer Res 2014; 74: 598-608.

38 Zubris KAV, Liu R, Colby A, Schulz MD, Colson YL, Grinstaff MW. In vitro activity of paclitaxel-loaded polymeric expansile nanoparticles in breast cancer cells. Biomacromolecules 2013; 14: 2074-82.

39 Jain S, Spandana G, Agrawal AK, Kushwah V, Thanki K. Enhanced antitumor efficacy and reduced toxicity of docetaxel loaded estradiol functionalized stealth polymeric nanoparticles. Mol Pharm 2015; 12 : 3871-84.

40 Bao Y, Guo Y, Zhuang X, Li D, Cheng B, Tan S, et al. D- $\alpha$-Tocopherol polyethylene glycol succinate-based redox-sensitive paclitaxel prodrug for overcoming multidrug resistance in cancer cells. Mol Pharm 2014; 11: 3196-209.

41 He Q, Gao Y, Zhang L, Zhang Z, Gao F, Ji X, et al. A pH-responsive mesoporous silica nanoparticles-based multi-drug delivery system for overcoming multi-drug resistance. Biomaterials 2011; 32: 7711-20.

42 Zhou Y, Yu Q, Qin X, Bhavsar D, Yang L, Chen Q, et al. Improving the anticancer efficacy of laminin receptor-specific therapeutic ruthenium nanoparticles (RuBB-loaded EGCG-RuNPs) via ROS-dependent apoptosis in SMMC-7721 cells. ACS Appl Mater Inter 2016; 8: 15000-12.

43 Liang D, Wang A, Yang Z, Liu Y, Qi X. Enhance cancer cell recognition and overcome drug resistance using hyaluronic acid and $\alpha$-tocopheryl succinate based multifunctional nanoparticles. Mol Pharm 2015; 12 : 2189-202.

44 Lv S, Tang Z, Zhang D, Song W, Li M, Lin J, et al. Well-defined polymerdrug conjugate engineered with redox and $\mathrm{pH}$-sensitive release mechanism for efficient delivery of paclitaxel. J Control Release 2014; 194: 220-7.

45 Dintaman JM, Silverman JA. Inhibition of P-glycoprotein by D- $\alpha$ tocopheryl polyethylene glycol 1000 succinate (TPGS). Pharm Res 1999; 16: 1550-6.

46 Tang L, Yang X, Yin Q, Cai K, Wang H, Chaudhury I, et al. Investigating the optimal size of anticancer nanomedicine. Proc Natl Acad Sci U S A 2014; 111: 15344-9.

47 Prabhakar U, Maeda H, Jain RK, Sevick-Muraca EM, Zamboni W, Farokhzad OC, et al. Challenges and key considerations of the enhanced permeability and retention effect for nanomedicine drug delivery in oncology. Cancer Res 2013; 73: 2412-7.

48 Niko Y, Arntz Y, Mely Y, Konishi Gi, Klymchenko AS. Disassemblydriven fluorescence turn-on of polymerized micelles by reductive stimuli in living cells. Chemistry 2014; 20: 16473-7.

49 Jess TJ, Belham CM, Thomson FJ, Scott PH, Plevin RJ, Gould GW. Phosphatidylinositol 3'-kinase, but not p70 ribosomal S6 kinase, is involved in membrane protein recycling: wortmannin inhibits glucose transport and downregulates cell-surface transferrin receptor numbers independently of any effect on fluid-phase endocytosis in fibroblasts. Cell Signal 1996; 8: 297-304.

50 Ly JD, Grubb DR, Lawen A. The mitochondrial membrane potential (deltapsi $(m)$ ) in apoptosis; an update. Apoptosis 2003; 8: 115-28.

51 Hrycyna CA, Ramachandra M, Ambudkar SV, Ko YH, Pedersen PL, Pastan I, et al. Mechanism of action of human P-glycoprotein ATPase activity photochemical cleavage during a catalytic transition state using orthovanadate reveals cross-talk between the two ATP sites. J Biol Chem 1998; 273: 16631-4.

52 Bansal T, Akhtar N, Jaggi M, Khar RK, Talegaonkar S. Novel formulation approaches for optimising delivery of anticancer drugs based on P-glycoprotein modulation. Drug Discov Today 2009; 14: 1067-74.

53 Choi SU, Lee CO, Kim KH, Choi EJ, Park SH, Shin HS, et al. Reversal of multidrug resistance by novel verapamil analogs in cancer cells. Anticancer Drugs 1998; 9: 157-65. 